Han Steenwijk

Sorbisches Institut/Serbski institut Cottbus/Chośebuz

\title{
The Contribution of Oroslav Caf to Resian Lexicography
}

Dedicated to the memory of Maria Di Lenardo-Barbarino Cúnkina

V članku je po abecednem redu predstavljenih več kot 300 rezijanskih besed iz Pleteršnikovega Slovensko-nemškega slovarja (1894-95), ki izvirajo iz zbirke Oroslava Cafa (1814-1874). Obravnavano gradivo je opremljeno $z$ dodatnimi tedanjimi in današnjimi narečnimi zapisi ter s kritičnimi pripombami.

The more than 300 Resian lexical items in Pleteršnik's Slovensko-nemški slovar (1894-95) for which Oroslav Caf (1814-1874) is credited are presented here as a separate list, accompanied by contemporary and modern attestations and some critical remarks.

\section{Introduction}

The following is intended as a tribute to the lexicographical and dialectological achievements of the self-taught linguist Oroslav Caf, who lived from 1814 to 1874 . $^{1} \mathrm{Al}-$ though the dictionary that he prepared never appeared in print, its materials were to contribute in a significant way to the lemmata in Pleteršnik's Slovensko-nemški slovar.

Caf was, among many other things, especially interested in Resian. He himself never visited Resia, but was still in a position to obtain first-hand information on the dialect, as a native speaker, Domenico Longhino, had settled in Fram, the village where Caf served as a curate. Judging by the epithet "Beljan" given to him by Caf, his informant probably originated from Bila/San Giorgio (Matičetov 1981-83: 235).

Apart from the lexicographical material which Caf extracted from Longhino over a period of 12 years of cooperation, ${ }^{2}$ we are also indebted to the industrious cleric for the text of two Resian songs, posthumously published by Milko Matičetov (1981-1983), and of an episode from the New Testament, published during his lifetime in Slovenska bčela (Caf 1852).

\section{Caf's Resian material in Pleteršnik}

The Resian data that are designated as such in Pleteršnik by the abbreviation $R e z$. originate from various sources. By means of additional abbreviations alongside Caf the following scholars are credited: Baudouin de Courtenay (72 lemma entries), Cigale (4 entries), Erjavec ( 2 entries), Klodič (8 entries), Levstik (4 entries), Miklošič (19 entries), Navratil (1 entry), Valjavec (8 entries) and Vraz (1 entry). Furthermore, for 5 entries containing the abbreviation Rez. the source has not been indicated. Apart from Jan Baudouin de Courtenay and Stanko Vraz the persons just mentioned never visited Resia and probably did not have first-hand knowledge of the

\footnotetext{
${ }^{1}$ For more detailed information on the life and work of Oroslav Caf, see Rudolf Kolarič, Caf Oroslav, Slovenski biografski leksikon 1, Ljubljana 1925-32, pp. 66-67.

${ }^{2}$ Thus Caf in a draft for a letter to Bleiweis (1861), quoted in Matičetov 1981-83: 234.
} 
dialect. $^{3}$ It is not only this fact that stresses the importance of Caf's contribution, but also the sheer number of the lemma entries in which he is credited: $329 .{ }^{4}$ Moreover, already a first glance at the Resian lexical units contained in them suffices to indicate that they are by no means trivial. Many of them are not known from other sources, either contemporary or preceding Caf's activities.

This state of affairs justifies the publication of the lexical units attributed to Caf as a separate list. The information in this list that is taken from Pleteršnik consists of the following elements:

a) the head word of the lemma entry, printed in bold type. When a Resian lexical unit or the specific Resian meaning being carried by the head word is mentioned only further on in the lemma as dialectal information, the head word is here given within parentheses, e.g., (bệl) belo 'das Splintholz' and (bẹlídica) 'das Wiesel'; 'schönes, schnippisches Mädchen';

b) the abbreviated indication on alternations and/or the flection class of the head word, also printed in bold type, e.g., brúmen -mna 'der Monat Jänner';

c) indications on the aspect and valency of verbs, e.g., brbískati if 'dristati' and fúkniti kam pf 'irgendwohin entflattern';

d) the translation, mostly in German, as in the above-mentioned examples except brbískati, sometimes in Slovene, as in brbískati;

e) when mentioned, a Resian dialectal form printed in italic type, as in the above-mentioned example (bẹ́l).

This section is closed by a full stop and reference to the volume and page number.

In order to set off Caf's contribution to our knowledge of the Resian lexicon against the information stemming from other sources up until 1900, attestations from such other sources are added. If an attestation can be found in the works of Baudouin de Courtenay, such a form is adduced to the exclusion of his precursors Dependent upon the nature of these sources the translations in this second section are in Russian, German, Slovene and Italian.

\section{Additional modern amendments to Caf's items}

However, some factors necessitate the addition of further information to this list. Firstly, Pleteršnik still remains, more than 100 years after its publication, an important source for lexical data on which other linguistic studies draw. This alone is already reason enough to check the items contained in it for their accuracy. in the case of data originating from Caf this seems no superfluous activity as he tended to etymologise in his transcriptions (Matičetov 1981-83: 241). Furthermore, Pleteršnik and his assistants are sure to have adapted dialectal items to the notation for Common Standard Slovene. Secondly, because of the considerable of time that has passed since Caf worked with his Resian informant it may be interesting to compare his lexicographical data with such that can be obtained in the field at the present stage of the dialect.

\footnotetext{
${ }^{3}$ On Miklošič as a secondary source for Resian data cf. Steenwijk 1992: 451-454.

${ }^{4}$ The 700 or so Resian lexemes that Matičetov (1981-83: 235) counted in Pleteršnik also include such without an explicit reference to Resian, but that can be attributed to the dialect because they are unknown elsewhere. This would then be a maximal list, against which ours is a minimal list of altogether 451 entries containing reference to Resian.
} 
Therefore the list is accompanied by modern Resian data mainly originating from two villages, Bila and Osoanë/Oseacco. The former village was the probable home village of Caf's informant and represents the western, more innovative dialect area, whereas the latter is representative of the eastern, more conservative area within Resian. Two ways of offering the lexical material to the female informants were used. First, a semantic description of an item was given, in Resian and/or in Italian, in order to elicit a reaction. When these reactions did not result in the naming of the probable Resian item that was noted by Caf, then Caf's notation was pronounced in one or more presumed dialectal ways and the informant was asked whether she was familiar with such and such a word. The informant for Bila was Maria Di Lenardo-Barbarino Cúnkina (1928-1999) and for Osoanë Anna Pusca Čẹkawa (1908-). In addition to a complete check in the afore-mentioned villages occasional and not systematically collected material from the villages of Njïwa/Gniva and Solbica/Stolvizza has also been included. The whole procedure of confronting the informants with Caf's data extended over several visits to the dialect area during the years 1992-1996.

The modern material is presented with the following conventions:

a) a division in two sections has been made. Information that follows immediately after the data taken from Pleteršnik is held not to conflict significantly with these data, neither from a formal point of view nor as regards the contents. In the case of apparent conflict between Caf and the modern data the latter are introduced by the remark "Cf., however," e.g., brệckati se if brecka se 'der Morgen dämmert'. I: 54. SG -; O -. Cf., however, O rré dín 'sta albeggiando';

b) the abbreviations SG for Bila, Lp for Lipovaz, G for Njïwa, O for Osoanë, $\mathrm{P}$ for Ravanca/Prato di Resia and $\mathrm{S}$ for Solbica introduce data originating from the respective local dialects;

c) the translations of the modern material originate from the informants and are therefore given in Italian;

d) our own findings are sometimes amplified with attestations by another researcher, Roberto Dapit, who collected his material more or less contemporaneously with this research.

After this section, closed by a full stop, sometimes remarks on various aspects of the information given in the lemma are made.

\section{Some general characteristics of the findings}

The probability that most of Caf's Resian attestations actually reflect the local variety of Bila is reinforced by the following peculiarity. The head words nûn, sûj and $\hat{\mathbf{u}} \mathbf{j}$, that within Resian show traces of an original ${ }^{*} \grave{u}$, are written with $\hat{\mathbf{u}}$. It is exactly the Bila variety where the opposition between $* \grave{u}$ and $* \bar{u}$ has been lost, as both reflect as ụ. Thus we have SG nụ́n, sụjj and wụj on one hand and wụt klubụ́k and wụm corresponding to hûd, klobúk and úm on the other. In the other Resian varieties this oppostion is reflected by $\dot{j}$ for $* \grave{u}$ and $\underline{u}$ for $* \bar{u}$, e.g., O nón, sój, wój against xụ̂t, klabụ́k, wụm. The erroneously standardised forms given in Pleteršnik are therefore most likely to have been based on material ultimately originating from a native speaker born and raised in Bila. 
One could object to this assertion that in the modern material sometimes no positive reaction from precisely the Bila informant was obtained. For several reasons this need not worry us at the present stage. Firstly, for the aim of this study generally no investigation into heteronymic relations was carried through. This means that pending further checking, involving more informants per village and specific confrontation with heteronyms, we can for the moment assume that at least in part such non-occurrence reflects individual language use. Secondly, the dialect lexicon of the 19th century is almost certain to have been different from the modern one. Then current lexemes may have been lost by now in one or more villages. This can be exemplified for the notion 'stockings'. Nowadays at Bila only škufóne can be attested for it, but in a text most probably written in the Bila variety and originating from the beginning of the 19th century we read laze [= láče $\leftarrow$ *hlače] (Riva 1966: 85). As this loss progresses slowly it can give rise to heteronymic relations that did not yet exist during Caf's times and that can be assumed to be responsible for another part of the cases of non-occurence.

The check with the modern language state was more or less successful in about half of the lexical units. Smaller deviations in form or content were by us still regarded as an affirmation of Caf's attestation, e.g., múla 'krava brez rogov' against SG múja, O múja 'capra senza corna' and even mîr 'bes. die Mauer ohne Mortel', although already during Caf's time exactly this notion is referred to by the lexeme SG mažérja 'muro a secco'. When both form and content showed slight deviations this was not regarded as a succesful attestation, e.g., hrípelj 'der Nasenscheidewandknorpel' against $\mathrm{O}$ xrịpavac 'cartilagine'. Leaving aside the relatively small group of lexical units for which only the etymon but not the relevant notion could be attested, we are confronted with a sizable number of cases (120 out of 329) in which not even the etymon mentioned in Pleteršnik's lemma entry was found.

The fact that many of Caf's Resian lexemes have not been registrated in other sources up until 1900 now turns out to be a mixed blessing. Out of these 120 cases only for vozíca 'der Schiebkarren' we have at least an additional attestation by Baudouin de Courtenay as G ûzica 'Schubkarren'.

As an explanation for the complete silence in the sources on the remaining 119 etymons only assumptions can be made. For some of them an attestation may be hoped for from the copious folk materials which Milko Matičetov collected in Resia during the whole of his professional career. These are now being transcribed in the Slovene Academy of Sciences and Arts.

When no further attestations will turn up, we seriously have to consider the possibility of language change and loss. An indication in this direction in the modern material is préja, for which the heteronymic relations have been checked. Assuming that Caf's informant was from Bila we conclude that this etymon must have been current there 150 years ago whereas nowadays out of all the four main varieties of Resian it is only to be found in Osoanë. If a large majority of these 119 etymons indeed represents language loss, than this loss is considerable and most alarming.

As in their standardised notation in Pleteršnik the actual Resian forms are not always readily recognisable (see the remarks to several entries in the list), the relevance of these 119 etymons for further linguistic analysis should in each individual case be carefully evaluated. For instance, among the etymons for which information from other 
sources is available, minor etymological corrections to the analysis as carried through in Bezlaj 1976- suggest themselves, cf. bêrčva, esej, etak, ezde, grúže and krósna.

\section{References}

Baudouin de Courtenay, Jan Ignacy

1875 Opyt fonetiki rezj'anskich govorov, Varšava-Peterburg.

1895 Materialien zur südslavischen Dialektologie und Ethnographie I. Resianische Texte, St. Petersburg.

1913 Materialien zur südslavischen Dialektologie und Ethnographie III. Resianisches Sprachdenkmal "Christjanske uzhilo", S.-Peterburg.

Bezlaj, France

1976- Etimološki slovar slovenskega jezika, 3 vols., Ljubljana.

Caf, Oroslav

1852 "Rozeansko i Ogersko-Slovensko naréčje”, Slovenska bčela 3, pp. 318-319, 399.

Dapit, Roberto

1995 Aspetti di cultura resiana nei nomi di luogo 1. Area di Solbica/Stolvizza e Korïto/Coritis, Padova.

1998 Aspetti di cultura resiana nei nomi di luogo 2. Area di Osoanë/Oseacco e Učjal Uccea, Padova.

Dobrovský, Josef $1834^{2}$ “Über die Slawen im Thale Resia”, in: idem (ed.), Slavin, pp. 118-124, Prag.

Faggin, Giorgio

1985 Vocabolario della lingua friulana, 2 vols., Udine.

Longhino, Antonio

1992 Val Resia: terra di arrottini, Udine.

Matičetov, Milko

1964 “'Pěsme rezianske' Stanka Vraza (1841)", Slovenski entnograf 16-17, pp. 203-215.

1981-83 “"Te dve ste rozeanski': Cafov zapis 1844”, Traditiones 10-12, pp. 233-244.

1989 "Lánita: un relitto lessicale paleoslavo", Metodi e ricerche n.s. 8/1, pp. 94-106.

1993 "Resia: 1. Dimensione linguistica", in: G. Fornasir \& G. P. Gri (eds.), La cultura po-

polare in Friuli: Lo sguardo da fuori. Atti del convegno di studio, Udine, pp. 57-94.

1998 "Iz rezijanske leksike", in: A. D. Duličenko (ed.), Jazyki malye i bol'šie: in memoriam acad. Nikita I. Tolstoi (= Slavica Tartuensia 4), Tartu, pp. 49-62.

Matičetov, Milko \& Gaetano Perusini

1955-56 "Un dizionaretto e due Paternoster resiani inediti", Ricerche Slavistiche 4, pp. $76-87$.

Pirona, Giulio Andrea et al.

1935 Il nuovo Pirona: vocabolario friulano, Udine.

Pleteršnik, Maks

1894-95 Slovensko-nemški slovar, 2 vols., Ljubljana.

Riva, Franco

1966 Tradizioni popolari venete secondo $i$ documenti dell'inchiesta del Regno Italico (1811) (= Istituto veneto di scienze, lettere ed arti: Memorie. Classe di scienze morali, lettere ed arti, vol. 34, fasc. 2), Venezia.

Sreznevskij, Izmail Ivanovič

1841 “O narěčijach slavjanskich”, Žurnal Ministerstva narodnago prosvěščenija 31/2, pp 133-164.

Steenwijk, Han

1992 "Miklošič als Resianologe", in: J. Toporišič et al. (eds.), Miklošičev zbornik (=Obdobja, vol. 13), Ljubljana, pp. 451-461.

1994 "Resian jsé, jtáku etc.: some etymologies reconsidered", in: A. A. Barentsen et al (eds.), Dutch Contributions to the Eleventh International Congress of Slavists. Linguistics (= Studies in Slavic and General Linguistics 22), Amsterdam, pp. 399-406. 


\section{The material}

badijân 'der Sternanis (illicium anisatum)'. I: 9.

$\mathrm{SG}-; \mathrm{O}-$.

bêč 'eine kleine Münze'. $I$ : 15.

P Apl béče 'den'gi' (BdC 1875: 36).

SG béč - $a$ 'moneta', Npl béčavi 'soldi'; O béč -a 'soldo', dạn valị́ke béč 'un soldo grande'; S béć 'moneta', ití ma béč 'ha tanti soldi'.

Remark: As a countable singular béč means just 'coin', not 'small coin'.

(bẹ́l) belo 'das Splintholz'. I: 17.

$\mathrm{SG}-$; O -.

(bẹlídica) 'das Wiesel; schönes, schnippisches Mädchen'. I: 18.

G Npl bilétice 'Eichhörnchen' (BdC 1895: 243, 568).

SG bilíidica/blídica 'donnola; persona dai capelli bianchi'; G bilética 'donnola'; O balídica 'donnola'; S balídica 'donnola'.

Cf., however, G Bilíídic (surname for a thin person).

Remark: G bilética can at least in part be explained as having been influenced by Fri. bilite 'donnola' (Pirona 1935: 55).

bẹloha 'weisse Ziege'. I: 19. $\mathrm{SG}-; \mathrm{O}-$.

bêrčva 'die Kohlpflanze'. I: 20.

P Npl b'éř̌we 'Krauskohl' (BdC 1895: 353).

SG béržwa 'verza'; O bérక̌wa 'verza'; S béržwa 'verza'.

Remark: The Resian forms contain a voiced affricate which coincides perfectly with Fri. verge [vèrğe] (Faggin 1985: 1558), cf. Bezlaj I: 17.

(brána) 'die Gitterthür'. I: 49.

O brân 'kalitka' (BdC 1875: 35).

SG brán - $a$ 'cancello (di ferro o di legno)'; O brán - $a$ 'cancello di legno; patta' (arch.), brayésæ ziz bránann 'pantaloni con una patta'; S brõn -a 'cancello; cerniera di pantaloni o di gonna'.

Remark: The Resian noun is a masculine, not a feminine.

brbískati if 'dristati'. $I$ : 52 .

SG -; O -.

Cf., however, SG drístat -šćẹn if 'avere la diarrea'; G prelsg drị́šcen if 'avere la diarrea'; O drístat -šćan if 'avere la diarrea', to mi dríśćce 'ho la diarrea'.

$\mathrm{SG}-; \mathrm{O}-$.

brệckati se if brecka se 'der Morgen dämmert'. I: 54 .

Cf., however, O rré dín 'sta albeggiando', yré búsk 'id.'.

brệsk 'die Morgendämmerung'. I: 55.

SG brísk -a 'alba', dén pušnụwa, ko jẹ brísk 'il giorno comincia, quando c'è l'alba'; O brísk 'alba'.

Remark: Isg pred breskon 'before dawn' (Caf 1852: 319) would imply that the noun once was subject to the alternation é $\sim i$, like Gsg lệda, svệta NAsg lit 'ice', svít 'world'. 
brúmen -mna 'der Monat Jänner'. I: 67.

$\mathrm{SG}-; \mathrm{O}-$.

Cf., however, SG brụman -ụwna/-ụ́na 'intraprendente, bravo, capace', క̌anár 'gennaio'; G brụ́man -ụ̂wna 'diligente', క̌anćr 'gennaio'; O brụmanan -ụ̂wna 'bravo', క̌anár 'gennaio'; S brụmen -ụ̂wna 'diligente', žanõr 'gennaio'.

Remark: As Caf's attestation is a loan from Friulian brume 'december' (Bezlaj I: 49), in Resian it probably had the form *brụma.

cẹ́ščenik 'der Schöps'. I: 81.

SG číšćanik 'uomo senza bambini’ (volg.); G číšćanik (said as an insult); O číšćanek 'uomo non fertile' (volg.); S číšćanik (said as an insult).

Cf., however, SG din číšćani mulón 'montone castrato; O ovan e číšćan 'il montone è castrato'; $\mathrm{S}$ ćaštrún 'montone castrato'.

Remark: Only the metaphorical meaning seems to have been preserved.

cŕkati if 'mucksen'. $I$ : 87.

SG cárkat -kan if 'farsi sentire; bere rumorosamente'; O cárkat non-O; S cárket, -ken if 'prendere gli avanzi di un liquido'.

Cf., however, O barbụlet 'fare rumore bevendo'.

Remark: The modern meanings that have to do with drinking confirm its onomatopeic origin as an imitation of the sound of dripping water (cf. Bezlaj I: 67).

číga 'die Lauer' na čigo iti, na čigo biti. I: 103. $\mathrm{SG}-; \mathrm{O}-$.

čígati if 'lauern, aufpassen' na koga čigati. $I: 103$.

SG čígat -gan if 'sbirciare; adocchiare', an čígal to hcị.. 'ha adocchiato la figlia', ja č́gan noga šlovẹka 'sto adocchiando un uomo'; O -.

čígav 'der gerne späht, lauert'. $I: 103$.

$\mathrm{SG}-; \mathrm{O}-$. $\mathrm{SG}-; \mathrm{O}-$.

čígavec -vca 'der Lauerer, der Spion'. I: 103.

čónkelj -klja 'dürrer Ast am Baume'. I: 108.

$\mathrm{SG}-; \mathrm{O}-$.

Cf., however, SG na womájana véja 'ramo secco sull'albero, senza corteccia'; O na omáana véa 'ramo secco senza corteccia'.

Remark: Cf. also girin and maybe SG čụnklin, S č̣̣nkej s.v. gúngelj.

črmnẹ̀l -ệla. I: 112 .

čerńèl 'krasnyj' (BdC 1875: 21).

SG čarnjẹl -alé 'rosso'; G čarnjẹl -alá 'rosso'; O čarnjệw -alá 'rosso'; S čarnjẹl -alá 'rosso'.

(čûh) 'der Gestank, der Modergeruch'. I: 116.

$\mathrm{SG}-; \mathrm{O}-$.

Cf., however, SG žmóh -óha 'sapore; puzza di putrefatto'; G jóh 'odore spiacevole’; O jóx -ụ̂xa 'odore di muffa, di umido'.

čúhniti if 'einen üblen Geruch, einen Modergeruch von sich geben'. I: 117. $\mathrm{SG}-; \mathrm{O}$-.

(dàr) dar do. I: 122.

dârdu 'do samogo, do samoj' (BdC 1875: 10). 
SG dárdu 'fin a'; G dárdu 'fin a', dárdu zụtra 'fin a domani'; O dárdu 'fin a'; a se šlá po nơáx dárdu rorẹ na Bríx 'sono andato a piedi fin sul Brich'; $\mathrm{S}$ dárdo 'fin a', dárdo won šíjo 'fin alla gola'.

(dệdec-dca) 'die Marionette'. I: 126.

dîdac 'deduška; čučelo, vypusknaja kukla' (BdC 1875: 84).

SG dídac - aca 'ometto; giocattolo nella forma di un uomino'; O dídac -aca 'piccolo uomo'; S dídec -aca 'uomo anziano'.

Remark: The e does not alternate with zero.

(dêdič) 'dedec 6. [der Haftelhaken]'. I: 126.

$\mathrm{SG}-; \mathrm{O}-$.

Remark: Such a meaning of the Resian lexeme would have been influenced by Ita. uomo (region.) 'sorta d'attaccapanni'. A Resian *dédić or *didić does not exist.

dohôjkati pf 'doklicati (kličoč: hoj!)'. I: 148.

$\mathrm{SG}-; \mathrm{O}-$.

domačîn 'der Hausgenoss'. I: 155.

$\mathrm{SG}-; \mathrm{O}-$.

Cf., however, G te hị̂šni ‘coninquilino’.

domačînka 'die Hausgenossin, die Einheimische'. I: 155.

$\mathrm{SG}-; \mathrm{O}-$.

drásiti if '(die Naht) auftrennen'. I: 167.

$\mathrm{SG}-; \mathrm{O}-$.

Cf., however, SG rizgnét 'disfare'; O riznát, rizdrít 'scucire'; S riznćt 'disfare'.

(drnǫ́hati) if "drnjuhati". 5 : 174.

SG darnjụ̣hat -šẹn if 'russare'; O darnjụ́xat -šan if 'russare'; $\mathrm{S}$ darnjụ̂wet -wen if 'russare'.

drózati if 'einsinken (im Koth, Schnee)' sneg se droza 'man sinkt im Schnee ein'. I: 177 .

ko j sníh, tu dróza 'wenn der Schnee ist, sinkt man ein' (BdC 1895: 385).

SG drózat -zan if 'andare e sprofondare; sprofondare fin ai ginocchi', ja drózan sník, lụžo; G pre3sg dróza 'essere morbido, cosicché si aprofondisca' (di neve), srín, ko an ni dróza sníh 'neve dura, quando la neve non fa sprofondare'; O drózat -za, sníx dróza 'la neve fa sprofondire', to dróza 'la gamba va dentro nella neve, nel fango; S drózet -za if, ja si šlá, ki me drózala zémja (when one is completely exhausted), ja si spál, da to je drózalo (when one is sleeping very profoundly).

Remark: The Resian verb is not reflexive.

esej, esa, eso '= ta' esej zde '= ta-le'. I: 197.

jisà 'èta' (BdC 1875: 75).

SG jsé, jsá, jsọo ‘questo’; O isí, isá, isọo ‘questo’.

Remark: Cf. on this and the following two lexemes Steenwijk 1994.

(etak) etako to bodi! '= amen!'. I: 197.

jtáku 'tak' (BdC 1875: 17).

SG jtáku 'così; O itáku 'così, itáku to bóde 'amen'.

${ }^{5}$ In Pleteršnik's material double quotes enclose forms that he cites more or less precisely according to the source. 
ezde 'tu-le'. I: 197.

jzdé 'zdes" (BdC 1875: 17).

SG jzdẹ 'qui'; O izdẹ 'qui'.

fârnik '= faran'. $I: 199$

$\mathrm{SG}-; \mathrm{O}$-.

fléra '= flara'. $I: 201$.

$\mathrm{SG}-; \mathrm{O}-$.

Cf., however, SG mágla' macchia'; O mágla 'macchia'.

fréča 'die Schleuder'. I: 202.

$\mathrm{SG}-; \mathrm{O}-$.

Cf., however, SG fjónda 'fionda'; O fréča 'freccia, fjónda 'fionda'; S smírjanjẹ 'fionda'.

Remark: Caf's attestation is probably influenced by Fri. frèzze (Pirona 1935: 346), Ita. freccia 'arrow'.

(fr̀k) na en frk so se ptiči razleteli. I: 203.

$\mathrm{SG}-; \mathrm{O}-$.

(fŕkati -kam -čem) if 'schnell werfen': drva v peč frkati. I: 203.

SG fárkat -kan -čẹn if 'lanciare'; O fárkat -kan -čan if 'buttare con forza', to mæ fárka 'sto traballando'.

fúkniti kam pf 'irgendwohin entflattern'. I: 204.

SG -; O -; S l-ptc msg fụ́knol 'comparire all'improviso'.

Cf., however, SG ufụkknut -ne pf 'comparire all'improviso', wtác jẹ ufụ́knul od gnjị$z d a$ 'l'uccello è comparso all'improviso dal nido', pás jẹ ufụknul nu wbížal 'il cane improvisamente compariva e scapava via', ni se bujịjo, ko wogínj ufụkkne 'hanno paura, quando il fuoco esce dal camino'; O vifụ́knut pf 'comparire all'improviso', wtéć je vifụ́knuw od rnjịzda 'l'uccello è comparso all'improviso dal nido'.

fúliti if 'abstreifen': perje, kožo fuliti; 'schleifend gehen'. I: 204. P fụ̂lyt hrèh 'die Erbsen abschälen, enthülsen' (BdC 1895: 53).

SG fụ́lit -lin if 'sbucciare, scortecciare' non-SG; 'spennare', fụ́lit kọ́kuš 'spennare la galina'; O fụlet -en if 'sbucciare, scortecciare; strappare (le penne)', fụlen bobịce 'sto sbucciando i fagioli'.

Cf., however, SG lụpit -in if 'sbucciare, scortecciare', lụpit kartụfule 'sbucciare le patate'; G na ufụjana krépa 'una testa calva'; O ofụlet plụmo od patalịna 'spennare il gallo; S ofụlit krampir 'sbucciare le patate'.

Remark: Outside Bila the primary notion referred to by the verb is more general than the one given in Pleteršnik. The metaphorical meaning, however, could not be confirmed.

gáda 'der Fehler, der Tadel': konj ima dve gadi. I: 205.

$\mathrm{SG}-; \mathrm{O}-$.

Cf., however, SG šklánt -ịnta 'difetto'; O difjẹt 'difetto'.

gâdba 'das Tadeln'. I: 205.

$\mathrm{SG}-; \mathrm{O}-$.

gadljìv 'scheußlich, hässlich'. I: 205.

$\mathrm{SG}-; \mathrm{O}-$. 
gáter -tra 'das Gatter, das Gitter'. I: 208.

O háter 'Drahtgitter' (BdC 1895: 274).

SG gátẹr-arja 'rete metallica'; $\mathrm{G}$ hátẹr 'rete metallica'; O yátẹr-arja 'rete’; $\mathrm{S}$ átẹr 'rete metallica'.

Remark: The e does not alternate with zero.

$\mathrm{SG}-; \mathrm{O}-$.

(gáziti) if gaziti vodo '= vodo bresti'. I: 208.

$\mathrm{SG}-; \mathrm{O}-$.

gir -rja ‘der Knorren’. I: 210.

Cf., however, SG čśkul -a 'nodo nel legno', gróp -ópa 'id.'; O yróp -ópa 'nodo nel legno'; S róp -ópa 'nodo nel legno'.

girin '= gir'. $I: 210$.

SG gị́rin - $a$ 'ramo secco; nodo di un ramo tagliato'; $\mathrm{O}$-.

gírjast 'knorrig': girjasto drevo; 'holprig': girjasta pot. I: 210.

$\mathrm{SG}-; \mathrm{O}-$.

gírjav 'knorrig'. I: 210 .

SG din gịrjavi járbul 'un'albero nodoso; $\mathrm{O}$-.

(gláva) 'der Hutgupf'. I: 213.

glâva 'golova' (BdC 1875: 116).

$\mathrm{SG}-; \mathrm{O}-$.

Cf., however, SG gláwa 'testa; cocuzzolo'; O rláwa 'testa'.

(glavína) 'der Hutgupf'. I: 214.

$\mathrm{SG}-; \mathrm{O}-$.

gliníšče 'die Thongrube'. I: 217.

$\mathrm{SG}$-; O -.

(gnèt -éta 'das Gedränge'): v cerkvi je bil gnet. I: 221.

$\mathrm{SG}-; \mathrm{O}$-.

Cf., however, SG to zamášćanu tu-w carkvé 'la chiesa è affollata’; O to bilu zarnátu judí 'era zeppo pieno di gente'.

$\mathrm{SG}-; \mathrm{O}-$.

gnẹtati -tam if 'drücken': gnetati koga. I: 221.

$\mathrm{SG}-; \mathrm{O}-$.

gnilína 'fauler Mensch'. I: 222.

Cf., however, SG an trášt 'è molto pigro'; O dan tréšt 'uomo pigro'.

(gnilóba) ‘= lenoba'. I: 222.

SG -; O - .

(gółcati) if 'rülpsen'. I: 227.

$\mathrm{SG}-; \mathrm{O}-$.

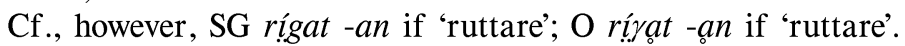

(gółčati) if 'tosen’ (o gromu). I: 227.

SG -; O -.

Cf., however, O to rarmị mọćnu 'sta tonando fortemente'. 
$\mathrm{SG}-; \mathrm{O}-$.

gồk 'das Tosen des Donners'. I: 228.

$\mathrm{SG}-; \mathrm{O}-$.

golọbec -bca 'der Adamsapfel'. I: 228.

SG golóbac 'pomo d'Adamo', ćon te jét za golóbac, ći ti ni mučç̌šs 'ti prenderò per la gola, se non starai zitto; $\mathrm{O}-$.

Cf., however, O ábuku ta-nu w ṣ̣̌̂e 'pomo d'Adamo'.

(goltáč) ‘der Kropf des Geflügels'. I: 229.

$\mathrm{SG}-; \mathrm{O}-$.

Cf., however, SG goltén -ána 'faringe'; O rowtắn -ána 'gola'.

Remark: Cf. also gọ́ža.

(goltáti) if 'rülpsen'. I: 229.

$\mathrm{SG}-; \mathrm{O}-$.

Cf., however, SG gúltat 'prendere un sorso'.

Remark: Cf. gólcati.

gomòt -óta 'das Gewühl in einem Haufen'; 'der Wirrwarr'. I: 231.

$\mathrm{SG}-; \mathrm{O}-$.

gorjàn 'der Gebirgsbewohner'. I: 233.

Npl Gộrjani 'gorskie žiteli, nom. propr.' (BdC 1875: 47).

SG gọ́rjan - $a$ 'montanaro delle Prealpi Giulie'; O rọ́rjan -ana 'abitante del comune di Torre'; S órjen -ana 'abitante del comune di Torre'.

Remark: In Resia not known as a common noun.

$\mathrm{SG}-; \mathrm{O}-$.

(gospodič) 'der Edelknabe, der Junker'. I: 236.

gostniti pf 'dicht werden'. I: 238.

SG-; O -

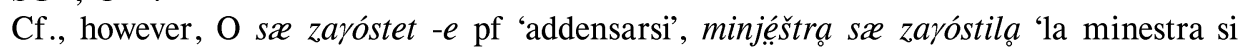
è addensata'.

(gọ́šča) ‘die Biestmilch’. I: 239.

SG góśća 'colostro'; O róšća 'deposito in un liquido'.

$\mathrm{SG}-; \mathrm{O}-$.

gozdọvski '= gozden'. $I: 242$.

Cf., however, SG gozdộwi 'del bosco’.

Remark: See also s.v. óvnovski.

gọža ‘der Kropf’; ‘der Hühnerkropf’. I: 242.

SG góža 'gozzo'; O róža 'gozzo'.

(grábiti) if vodo grabiti ' $=$ zajemati'. I: 242.

grábit 'grabit', napr. sěno' (BdC 1875: 44).

SG -; O -.

Cf., however, SG grábit -in if 'rastrellare', grábit tarénj 'rastrellare il terreno', grábit $d w$ oọr 'rastrellare il cortile'; G hrábit 'rastrellare'; O rrábet -en if 'rastrellare', rrábet sẹnu, rráwo 'rastrellare il fieno, il ghiareto'. 
grâsp 'die Klaue, die Kralle'. $I$ : 245.

SG Apl gráspe 'Klauen, Krallen’ (BdC 1895: 19).

SG gráspa 'artiglio; zampa'; O -.

Cf., however, O párkej -kja 'artiglio; zampa'; S párkej 'zampa'.

Remark: The Resian noun is a feminine, not a masculine.

grâspati -pam if '= praskati'. I: 245 .

SG gráspet 'kratzen' (BdC 1895: 526/14).

SG gráspat -pan -pjẹn if 'graffiare'; G hráspat -pjẹn 'graffiare'; O rráspat -pan, - pjąn if 'graffiare'; S ráspet - pjẹn 'graffiare'.

$\mathrm{SG}-; \mathrm{O}-$.

(ǵŕca) ‘der Brocken’: grča mesa. I: 247.

(grdína) 'das Gespenst'. I: 247.

SG gardína ‘drakon' (BdC 1875: 64).

SG gardịna 'persona femminile mitologica molto brutta'; O rardịna (said as an insult to an ugly person).

$\mathrm{SG}-; \mathrm{O}-$.

greblọ ‘= greblja, grebljica’. I: 249 .

$\mathrm{SG}-; \mathrm{O}-$.

gŕgavec -vca ‘die Gurgel'. I: 251.

Cf., however, O rowtán -ána 'gola'.

grîgec -gca 'die Grille'. $I: 251$.

O rríicic 'Grille' (BdC 1895: 577/291).

SG grígić -ića 'grillo'; G hrịhić 'grillo'; O rríreć -ića 'grillo'.

Remark: The Resian forms correspond to grîgič, not grîgec.

grigetáti -etâm/-éčcem if 'zirpen': grigič grigeče; 'klingeln'. I: 251.

G hrihićát 'schwirren' (BdC 1895: 199).

SG grigićét -ćá if 'cantare (da grilli); sonare', grígić grigića 'il grillo sta facendo il suo verso; il sonaglio suona'; O rriyićát -ćán if 'sonare', yríyeć yriyićá 'il campanello suona'.

Cf., however, O púọ rríriće 'i grilli cantano’.

(grîgič) 'die Schelle'. I: 251.

O rríić 'Schelle' (BdC 1895: 577/291).

SG grígić -ića 'sonaglio; O rrị̂eć -ića 'campanello chiuso'.

(gr̂m) 'die Haselnussstaude'. I: 253.

hàrm 'kust, orěšina, leščina' (BdC 1875: 14).

SG górm - $a$ 'nocciolo'; O rórm - $a$ 'nocciolo'.

(grmáda 'der Haufen’) grmada drv. I: 254.

$\mathrm{SG}-; \mathrm{O}-$.

Cf., however, O dan kóp dárw 'un mucchio di legno'.

Remark: Cf. also tâsa.

$\mathrm{SG}-; \mathrm{O}-$.

grota 'der Leiterwagen'. I: 256.

grúžav '= garjav'. I: 259.

$\mathrm{SG}-; \mathrm{O}-$.

Cf., however, O rúšsjuw 'scabbioso'. 
$\mathrm{SG}-; \mathrm{O}-$.

grúže ‘= garje’. $I: 259$.

Cf., however, SG rụ́ša 'rogna'; G rụ́ša 'rogna'; O rụ́ša 'scabbia'; S rụ́ša 'scabbia'.

Remark: This necessitates a rethinking of the lemma grúže (Bezlaj I: 184). Maybe a link with rúša 'vrhnja plast zemlje z rastlinjem' (Bezlaj III: 209) exists.

(gúngelj-glja) 'der Finger- oder Fussknöchel'. I: 261.

SG -; G hụ̂ngaj 'malleolo'; O rụngej -gja 'malleolo'.

Cf., however, SG čụnklin - $a$ 'nocca', šlẹn - $a$ 'caviglia, malleolo'; O člẹn 'nocca'; S č̣̣nkej 'nocca', člẹnn 'caviglia'.

gungljáti -âm if 'mit grossen, mageren Füssen einhergehen'. I: 261.

$\mathrm{SG}-; \mathrm{O}-$.

hę̂k 'der Chorgesang'. I: 265.

$\mathrm{SG}-; \mathrm{O}-$.

Cf., however, SG ékat -čẹn if 'cantare in chiesa'; O rékanje 'il cantare in chiesa'.

Remark: Unexpected is the initial voiced fricative in this and the following Oseacco attestation.

(hînkati -kam/-čem) if bolnik hinče 'ächzt'. I: 267.

SG jínkat -če if 'lamentare'; O rịnkat -čan if 'gemere'; $\mathrm{S}$ inket -čajo if 'gemere'.

hláčica 'die Socke'. I: 269.

SG -; O xláčica 'calzino'.

Cf., however, SG kalcịn 'calzino; O tæ krátkæ xláčæ 'calzini' (come componente del costume tradizionale resiano).

(hlópniti) pf pes meso hlopne in požre. I: 273.

$\mathrm{SG}-; \mathrm{O}-$.

Cf., however, O pás popáduw mísu 'il cane ha afferrato la carne'.

(hrást) bukov hrast '= bukev'. I: 279.

hrâst 'listvennoe derevo' (BdC 1875: 26).

SG rást $-i /-\hat{\varepsilon}$ 'fusto del faggio'; G hrást - $\hat{\varepsilon}$ 'fusto del faggio'; O na bụ́kawa xrást 'un faggio', rrást -á 'faggio; fusto dell'albero' (Dapit 1998: 72); S rást -i/-é 'faggio'.

$\mathrm{SG}-; \mathrm{O}-$.

(hrípelj -plja) 'der Nasenscheidewandknorpel'. I: 282.

Cf., however, G hrịpovac 'cartilagine'; O xrịpavac -uwca 'cartilagine; cista'.

(hûd) huda roka '= leva roka'. I: 286.

G hụda róka 'schlimme Hand, linke Seite' (BdC 1895: 154).

SG wụ̂t $-d a$ 'cattivo; sinistro', ta wụ́da róka 'la mano sinistra'; G hụ́t 'cattivo, violente; sinistro'; O xụt 'cattivo; sinistro', ta xụ́da róka 'la mano sinistra’; $\mathrm{S}$ na to ụdo róko 'sul lato sinistro'.

(húdec -dca) 'der Zaunkönig'. I: 286.

O hụ́dac 'Zaunkönig' (BdC 1895: 286).

SG wụdac -aca 'scricciolo'; O xụdac -aca 'scricciolo'; S ụ́dec -aca 'scricciolo'.

Remark: The e does not alternate with zero.

(hûk) 'das Geräusch'. I: 288.

$\mathrm{SG}-; \mathrm{O}-$.

Cf., however, SG batabụjj - $a$ 'chiasso'; O šušụ̂r -rja 'chiasso'. 
(hûkati -kam/-čem) 'lärmen, schreien'. I: 288.

$\mathrm{SG}-; \mathrm{O}-$.

itam '= tam-le'. I: 299.

G jitàn 'tuda' (BdC 1875: 37).

SG jtén 'là'; O itạ́n 'là'.

itu 'gerade hier'. I: 299.

jitò 'tam' (BdC 1875: 57).

SG jtú 'lì'; O itó 'lì'.

iv '= ivje, inje'. $I: 299$.

$\mathrm{SG}-; \mathrm{O}-$.

Cf., however, O slána 'brina'.

izvrtína 'das Bohrmehl'. I: 352.

$\mathrm{SG}-; \mathrm{O}-$.

(jáma) 'das Loch'. I: 357.

jáma 'jama' (BdC 1875: 16).

SG jáma 'buco; grotta'; G jéma 'tana'; O áma 'buco; tana'; $\mathrm{S}$ jõma 'buco; tana'.

jệda '= ajda'. $I: 362$.

P jẹjda 'Buchweizen' (BdC 1895: 45).

SG jêjda 'grano saraceno'; O êjjda 'grano saraceno'; $\mathrm{S}$ jéjda 'grano saraceno'.

jerov '= jerej" pl “jerove”. I: 367.

jẹrö 'svjaščennik' (BdC 1875: 17).

SG jẹ́ru -ra 'prete', Npl jẹravi; G éru -ra 'prete', Npl ẹ́ruvi; O éruw -ra 'prete', $\mathrm{Npl}$ ẹrave; S Npl jẹravi 'prete'.

Remark: The final $\mathbf{v}$ is the result of an erroneous reconstruction (Matičetov 1993: 81, foot-note 107).

(kâmba) 'der Kinnbacken'. I: 382.

SG kámba 'hölzernes Halsband' (BdC 1895: 362).

SG kámba 'mandibola'; $\mathrm{O}$ kámba 'mandibola'; $\mathrm{S}$ kõmba 'metà della mandibola'.

(kâmenje) 'eine Bergstaudenfrucht'. I: 383.

kámańe 'moroška' (BdC 1875: 98).

SG kámanjẹ 'variante del mirtillo'; O -; S kòmanjẹ 'Rubus saxatilis' (Dapit 1995: 98).

Cf., however, O Kámanjẹ TOP.

kamōča 'die Gemse'. I: 384.

P ćamúrče Npl 'Gemsen' (BdC 1895: 61).

SG ćamúrča 'camoscio'; $\mathrm{G}$ ćamúrča 'camoscio; O ćamúrča 'camoscio'; S ćamúrča 'camoscio'.

(klobúk) 'eine Art grosser Schwamm'. I: 411.

O klöbụ́k ‘šapka' (BdC 1875: 96).

$\mathrm{SG}-; \mathrm{O}-$.

Cf., however, SG klubụ́k -a 'cappello; O klabụk 'cappello'.

(2. kò) ko teci 'komm nur'. I: 415.

SG kọ́j 'solamente', kọj tacá! 'vai, vai!', koj prịdi! 'vieni pure!; G kọj 'soltanto’; O koj 'solamente', kọj prị́de! 'non mancare a venire!'; S kọj ‘soltanto’. 
(kòł) 'der Zaunpfahl'. I: 420.

kọ́l 'kol, palka' (BdC 1875: 49).

SG kọ́l -olé 'randello, bastone'; O kộw -oláa 'palo per recinto; palo di sostegno'.

(kòłk) 'der Hügel'. $I: 424$.

G kólka Gsg 'Hügel' (BdC 1895: 137).

SG kúlk - $a$ 'collina'; G kólk 'collina'; O kúk 'collina'.

koredica '= red'; 'der Grasschwaden (beim Mähen)'. I: 437.

SG korédice Apl 'Heu-Reihen' (BdC 1895: 20, 587).

SG korédica 'andana di fieno'; G Apl kurédice 'andana di fieno'; O korédica 'lunga fila d'erba falciata'.

(koščíca) '= kocka', 'der Würfel'. I: 445.

O košćíca 'kostočka' (BdC 1875: 94).

$\mathrm{SG}-; \mathrm{O}-$.

Cf., however, SG kušćịca 'ossicino’; O košćịca 'osso piccolo'.

košet '= kozel'. I: 446.

kušệte Apl 'Böcke' (BdC 1895: 381).

SG kušẹt - $a$ 'caprone'; G kušẹt 'caprone'; O kušẹt - $a$ 'caprone'; S kušét 'caprone'.

Remark: The uniformity of the attestations leaves no doubt that $\mathbf{o}$ should be $\mathbf{u}$.

1. krek 'der Haken'. I: 462.

G krékove Npl 'krjuki' (BdC 1875: 80).

SG krék -éka 'gancio; rocca'; G krék 'gancio'; O krék -éka 'gancio'.

(krę̂lvlja) 'die Radfelge'. I: 465.

$\mathrm{SG}-; \mathrm{O}-$.

Cf., however, SG kréwlin -a 'uomo zoppicante'; G kréwja 'legno curvato per fare una slitta' (Dapit 1998: 88); O kréwja 'pezzo di legno curvato', ni so naréale žlịkæ ziz kréwjo 'facevano slitte con un legno curvato', șịna od kọla 'cerchione'.

2. krīpa ‘der Fels, der Steinklumpen'. I: 468.

cripa 'pietra' (Matičetov \& Perusini 1955-56: 84).

SG krápa 'grande pietra isolata'; $\mathrm{O}-$.

Cf., however, SG krépa 'testa', ćon ti dét dọ po krépi 'ti picchierò per la testa'; $\mathrm{O}$ krépa 'roccia su un pendio; testa'; $\mathrm{S}$ krépa 'coccio, testa, pietra (tombale)' (Dapit 1995: 118).

Remark: These are two etymons, *krịpa and krépa (cf. Bezlaj II: 93, 89). The unusual accented vocalism in SG krápa can be explained as an internal loan in which Bila $a$ renders the phonetics of $\underline{i}$ in the other local dialects.

2. krīpica 'das Steinchen'. I: 468.

SG krápica (dim); O -.

Cf., however, SG krépica 'testa' (dim.); O krépica 'testa' (dim).

krketjáti if '= krkljati'. I: 472.

SG karkaćét (said when polenta is coming to the boil) non-SG; O karkaćát -á if 'gorgogliare', múčnek karkaćá 'la polenta molle sta gorgogliando'.

kroketjáti if 'quacken': žaba kroketja. I: 475.

SG krokoćét -ájo if 'gracidare (dei rospi)'; O -.

Cf., however, O žába kwáče 'la rana sta gracidando'. 
krósma '= krošnja'. $I: 476$.

crosma 'ein Z-artiger Tragbalken, welcher zum Tragen der Waaren auf dem Rücken dient' (BdC 1895: 382, foot-note).

SG krọ́sma 'strumento per arrotare coltelli'; G krọ́sma 'strumento degli arrotini'; O krọ́sma 'strumento degli arrotini'.

Remark: This instrument, not in use anymore, was carried on the back or mounted on wheels, see Longhino 1992. With the attested meaning the Resian lexeme is now semantically closer to krosna than to krošnja (cf. Bezlaj II: 100).

$\mathrm{SG}-; \mathrm{O}-$.

krosmár -rja '= krošnjar'. I: 476 .

Cf., however, Lp krámar 'venditore ambulante'; S krõmer -arja 'venditore ambulante'.

2. krúcelj -clja ‘der Eiszapfen’. I: 480.

SG -; O krúcej -cja 'ghiacciolo’.

2. kuc 'die Welle'. $I: 484$.

SG -; O wọ́da na naréa næ valịkæ kụ́cæ 'lacqua sta facendo delle onde grandi'.

Cf., however, SG kụ́c 'capriola', si dál din kụ́c 'ho fatto una capriola'.

kúcati se -cam/-čem if 'Wellen schlagen': morje se kuca (kuče). I: 484.

$\mathrm{SG}-; \mathrm{O}-$.

Cf., however, SG se kúcat -an if 'fare capriole'.

kukûcati if 'kukati'. I: 486.

SG kukụcat -an if 'cuculiare; spiare'; O -.

Cf., however, G pre3sg kukụwa 'cuculiare'; O kukụcat -an if 'spiare', kukụwat -a if 'cuculiare'.

kukuríkati -kam/-čem if 'krähen (vom Hahn)'. I: 486.

pre3sg kukurî̌ce 'kukurekaet' (BdC 1875: 52).

SG kukurịkat -čẹn if 'cantare (dei galli)'; O kukurịkat -če if 'cantare (dei galli)'.

kultra '= kolter' konjska kolter 'die Schabracke'. I: 486.

SG kụltra 'coperta pesante; copriletto'; O kụ́wtra 'coperta imbottita'.

Remark: For horses a kind of blanket named kóca was used.

kúzelj -zlja ‘der Hund'. I: 491.

$\mathrm{SG}-; \mathrm{O}-$.

(laníta) ima črnele lanite. I: 499.

Lpl lanitah 'licih' (Matičetov 1964: 210, 211).

SG lánita 'guancia'; O lánita 'guancia'; S lõnita 'guancia'.

Remark: Cf. for this lexeme Matičetov 1989. Stress is unequivocally located on the first syllable.

lapúča pogl. lopuča. I: 500.

SG lipựca 'asse lungo con un manico per sbattere il sapone dal bucato; O lopứča 'arnese rettangolare di legno con un manico che serve a battere roba di lana o tela di lino'.

(lasníca) 'eine Art langes Gras'. I: 500.

SG lasníca 'una specie d'erba'; O -.

Cf., however, S lasina 'erba secca e gelida'. 
legâvec -vca '= lažnivec'. I: 506.

SG lágavac -awca 'bugiardo’; G láhovac -uwca 'bugiardo; O láravac -uwcà 'bugiardo'.

$\mathrm{SG}-; \mathrm{O}-$.

1. lihati if 'verschlucken'. $I: 517$.

Cf., however, SG to mu šlọ wprík 'gli è andato di traverso'; O to mi sæ wstávilu $t u$ - $w$ rowtáne 'mi è andato di traverso'.

lízgati se -gam if 'sich schminken'. I: 524.

$\mathrm{SG}-; \mathrm{O}-$.

Cf., however, SG se parkjét -án if 'truccarsi; sporcarsi', na se parkjá wụ́n po vị́šti 'si sta truccando la faccia'; O sæ bárbat 'truccarsi'.

(ljúbiti) if 'liebkosen, herzen'. I: 524.

$\mathrm{G}$ jụ́bit if 'accarezzare'; O jụbet -en if 'accarezzare'.

Cf., however, SG bújit -in if 'accarezzare'.

(2. mášča) 'die Obstmaische' "mešča”. I: 555.

SG mášća 'mosto'; O -.

Cf., however, O méšta 'mistura di patate, fagioli e altre verdure'.

medovje 'die Mistel (viscum)'. I: 563.

$\mathrm{SG}-; \mathrm{O}-$.

Cf., however, SG vášk -ị̂ška 'vischio'; O véšk -ị̂ška 'vischio'.

(2. mîr) 'bes. die Mauer ohne Mortel, z. B. zur Einfriedung'. I: 584.

m'îr 'stena' (BdC 1875: 18).

SG mír - $a$ 'muro (in senso generico)'; G mír 'muro'; O mír - a 'muro', xị̂ša e náret ziz míran 'la casa è fatta con un muro', mír e náret ziz cimíntan 'il muro è fatto con cemento'; $\mathrm{S}$ mir 'muro'.

Cf., however, SG mažérja 'muro fatto senza malta lungo la strada, muretto'; O mažérjạ 'muro a secco' (Dapit 1998: 108).

Remark: As already Baudoiun de Courtenay (1895: 213) has G mažẹrja 'eine Mauer, gemacht ohne Kalk', the information given by Pleteršnik seems to be the result of some misunderstanding.

2. míriti if '= zidati'. $I: 584$.

$\mathrm{SG}-; \mathrm{O}-$.

Cf., however, SG ja naréjan din mir 'sto costruendo un muro'; O a naréan dạn mír 'sto costruendo un muro'.

mit 'die Stange, der Pfosten, die Säule'. I: 586.

G m'it 'stanga' (BdC 1895: 136, foot-note).

SG mít $-i$ 'paletto lungo, usato per costruire un recinto'; G mít 'stanga'; O -.

Cf., however, O láta 'eine Stange im Zaune' (BdC 1895: 275), látạ 'pertica, stanga' (Dapit 1998: 97); S láta 'stanga'.

Remark: Judging by Caf's and Baudouin de Courtenay's attestations, the distribution of these heteronyms has remained stable over the past 150 years.

(2. morec -rca) 'schwarzer Bock'. I: 602.

$\mathrm{SG}$-; $\mathrm{O}$-.

moríca 'schwarze Ziege'. I: 602.

G móra 'eine schwarze Ziege' (BdC 1895: 186). 
SG móra (name for a black cow); O -.

Cf., however, SG múrica 'mora'; O múrica 'mora', na čárna taj múrica ‘è nera come una mora'.

Remark: It remains unclear whether Caf's attestation is actually the denomination of a fruit used as the name of a goat or a diminutive of the attested proper noun.

(moríža) moriže 'die Halskrause'. I: 602.

SG murị̌ze 'frangia'; O morížze -rí:s 'frangia'.

motíkati if 'mit der Haue hauen'. I: 604.

G mutikàt 'mit einer Hacke, Haue, Karste graben' (BdC 1895: 217).

SG mutikét -án if 'zappare'; O mutikát -án if 'zappare'.

motíne 'der Bodensatz einer Flüssigkeit'. I: 605.

$\mathrm{SG}-; \mathrm{O}-$.

mudžol pogl. mužol. I: 616.

P muక̌úl 'Glas' (BdC 1895: 55).

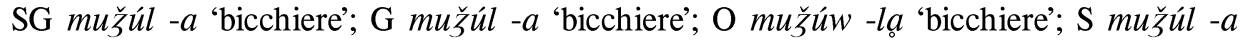
'bicchiere'.

(2. múla) 'krava brez rogov'; 'die Wurst'. I: 618.

G mụ́ja 'eine Ziege, welche keine Hörner hat' (BdC 1895: 186), mụ́lica 'Darm' (BdC 1895: 361).

SG múja 'capra senza corna'; múlica 'pezzo di intestino', múlice -cuw 'intestini'; G múlice 'intestini'; O múja 'capra senza corna', to taj na mụja 'manca qualcosa', múlicæ -lec 'interiora'; S múja 'capra senza corna', múlica 'pezzo di intestino'.

Cf., however, SG klobasịca 'salsiccia'; G klobasị́ca 'salsiccia'; O klabasịca 'salsiccia'; S klabasịca 'salsiccia'.

(mûlič) 'der Teufel' (šaljivo). I: 618.

SG mụjić - $a$ 'capretto senza corna; diavolo'; O mújeć -ića 'capretto senza corna; diavolo'.

múša '= oslica', Mez.-C. I: 621 .

P múšsa 'Eselin' (BdC 1895: 55).

SG múša 'asino femminile'; O -.

Remark: As the abbreviation Mez. is otherwise not used in Pleteršnik, I assume it is a printing error for $\mathrm{Rez}$.

múzgalọ 'das Maul'. I: 621.

muzgalö 'guba' (BdC 1875: 24).

SG múzzgalu 'labbro; labbra'; G múzzalu 'labbro; labbra'; O múzzgalu 'labbro; labbra'; S múzzalọ 'labbro'.

(múžiti) if 'etwas Weiches kauen, essen': hruške mužiti. I: 622.

SG múždet -en if 'masticare qualcosa di morbido', múžden din kós krụwa 'sto masticando un pezzo di pane'; O múzgat -ždạn if 'masticare qualcosa di morbido'.

mužôl 'das Trinkglas'. I: 622.

P mǔ̌úl 'Glas' (BdC 1895: 55).

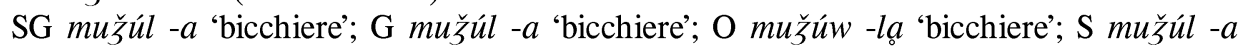
'bicchiere'. 
$\mathrm{SG}-; \mathrm{O}-$.

nadẹlováti if impf. ad nadelati: nadelovati pot, cesto. I: 630.

Cf., however, SG ni díwajo gráwo ta-na pót 'stanno mettendo ghiareto sulla strada'.

nárus 'röthlich'. I: 664.

SG nárus 'marone chiaro', an je nárus 'è di colore marone chiaro'; O nárus 'tendendo al giallo', tọ ẹ nárusu 'tende al giallo'.

Remark: Resian SG rús, G, O, S rós covers colour shades ranging from yellow through brown. For the notion 'red', see črmnẹ̀l

2. naváditi pf '= ovaditi'. $I: 676$.

O ji nawádeu 'belehrte sie' (BdC 1895: 256).

SG nawádit -in pf 'palesare; denunciare, riferire', ja ćon wan nawádit no ríč 'Le voglio render noto una cosa', mórrẹn tit ga nawádit 'posso andare a denuciarlo; $\mathrm{O}$ nawádet -en pf 'denunciare, fare la spia', an nawádew varatát 'ha raccontato la verità', nján ćon te nawádet 'adesso dirò la verità su di te'.

2. navájati if impf. ad navaditi '= ovajati'. I: 676.

SG nawájat -an if 'denunciare, riferire'; O nawáat -an if 'denunciare, fare la spia'.

nèr '= nego', 'sondern': ne kruh, ner voda. I: 699.

$\mathrm{SG}-; \mathrm{O}-$.

Cf., however, SG si nị láčan krụ́wa, si žéjan wodé 'non sono affamato di pane, ma assetato di acqua'.

nocọ́ ‘= nocoj’. I: 713 .

nacô 'segodnja večerom' (BdC 1875: 62).

SG nacó 'stanotte'; G nacó 'stanotte'; O nacó 'stanotte'; S nacó 'stanotte'.

nor '= enkrat' (Vestnik II: 61). I: 715.

nûr 'odin raz' (BdC 1875: 10).

SG núr 'una volta'; G núr 'una volta'; O núr 'una volta', se klị́cuw koj núr 'ho gridato solo una volta'; $S$ núr 'una volta'.

nûn '= nunec', 'der Taufpathe'. I: 719.

nòn 'kum' (BdC 1875: 57).

SG nụ́n -a 'padrino; signore’; G nón -ụ́na 'santolo; signore; O nón -ụ́na 'padrino; uomo qualunque'.

nuvìc ' $=$ novic 2. ' I: 720.

SG nuvič 'ženix' (BdC 1875: 60).

SG nuváč -liča 'sposo'; O novéč -ịča 'sposo’.

Remark: The unstressed vowel should rather be $\mathbf{o}$.

SG -; O -.

odprẹ́da '= od spredaj'. $I: 781$.

odpréjiti pf 'aufschnallen': odprejiti oblačilo. I: 781.

$\mathrm{SG}-; \mathrm{O}-$.

Cf., however, SG wođát rịnćo 'sfibbiare'; O odát préo 'sfibbiare'.

odumẹ́ti se -mím pf 'sich auf den Ruf melden'; 'aus dem Schlaf, aus der Betäubung (infolge Rüttelns, Rufens) zu sich kommen'. I: 791.

G ni so udumẹly ọ́badwá 'beide kamen wieder zu sich' (BdC 1895: 122).

SG udumẹt -ijẹn pf 'appena percepire', udumẹt din glás 'appena percepire un suono'; 
O sæ odumẹt -ín pf 'riaversi dal sonno'.

Cf., however, SG se wotpomanụt -ện pf 'riaversi dopo lo svenimento'.

$\mathrm{SG}-; \mathrm{O}-$. ogâba 'der Ekel'. I: 796.

(ogràd) vinski ograd 'der Weingarten'. I: 802.

SG Wogrét -áda TOP (small mountain); O -.

Cf., however, O orrádak -tka 'pezzo di terreno dove il fieno è messo assieme'; Lp orráda 'fieno messo assieme'.

(ogrâja) 'der Damm, der Deich'. I: 803.

$\mathrm{SG}-; \mathrm{O}-$.

(opộrek -rka) 'ein Lappen, am Schuh, woran die Schnalle oder die Schnur befestigt ist'. I: 839 .

SG -; G upúrak 'tomaia'; O opúrąk -rka 'pezzo di cuoio sullo zoccolo'; S Npl opúrke 'tomaia'.

(opr̂stnik) ‘= podplat', 'die Schuhsohle’. I: 844.

$\mathrm{SG}-; \mathrm{O}-$.

osójenica 'neka gibanica iz koruzne moke', 'abgeschmalzene Polenta'. I: 858.

SG wosójanica 'piatto fatto con polenta, ricotta e panna'; O osónica 'polenta molle con latte, formaggio e burro fuso.

$\mathrm{SG}-; \mathrm{O}-$.

ostẹja 'die gemauerte Seitenwand am Feuerherd oder am Kamin'. I: 861.

(ostróga) 'der Brombeerstrauch (rubus fruticosus)'. I: 863.

$\mathrm{SG}-; \mathrm{O}-$.

Cf., however, SG rubịda 'rovo'.

óvnovski 'Widder-, Schöpfen-'. I: 877.

$\mathrm{SG}-; \mathrm{O}-$.

Cf., however, SG Nsgf mulónawa 'di montone’; O ownộwje 'di montone’.

Remark: Cf. for the same desubstantive suffix -ộwj- SG gozdộjji s.v. gozdọvski and $\mathrm{O}$ konjộwje s.v. párkelj, in all three cases used to derive a relational adjective from an accent class (b) substantive.

3. pâl 'das Ziel': $v$ pal streljati. II: 4.

SG -; O -.

(palûd) '= rogoz'. II: 6 .

SG palụt - $d i$ 'canna palustre'; O palụt - $d a$ 'palude'; 'pianta non identificata' (Dapit 1998: 129), roré po Palúdu TOP.

panola pogl. panogla. II: 7.

Lp panúla 'Kolben vom Mais' (BdC 1895: 356).

SG panúla 'panocchio di granoturco'; G panúhla 'panocchia di granoturco; $\mathrm{O}$ panúla 'panocchio'; S panúla 'panocchio di granoturco'.

(párkelj-klja) 'der ungespaltene Huf', '= kopito'. II: 9.

párkaj 'kogot', kopyto' (BdC 1875: 19).

SG párkja 'zampa'; G an ma dólhe parkje 'ruba'; O párkej -kja 'artiglio; zampa', te konjộwje párkej 'lo zoccolo del cavallo; S párkej 'zampa'. 
(pệč) 'der Stein' “peć”; 'der Kummer, der Gram'. II: 16.

péćckamen” (BdC 1875: 19).

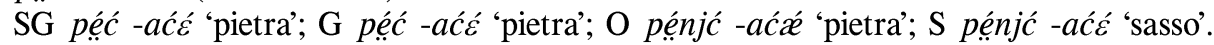

(1. pęéča) ‘der Gram’: veliko pečo imeti. II: 16.

$\mathrm{SG}-; \mathrm{O}-$.

Cf., however, SG péća 'mancanza nel carattere', to škọda, ki an ma to péco za se díwat klét 'peccato che ha quel difetto di mettersi a sgridare'; $\mathrm{G}$ péća 'difetto fisico o mentale'; O péća 'male fisico'.

Cf., however, SG faštị̂bi 'pena'.

(pečár -rja) 'tisti, ki peči kreše; der Steinhauer'. II: 16.

$\mathrm{SG}-; \mathrm{O}-$.

(péčen -čna) 'kummervoll': vsa pečna sem te iskala. II: 17.

$\mathrm{SG}-; \mathrm{O}-$.

(pečęen) ‘= kamenit', 'aus Stein': pečena miza. II: 17.

$\mathrm{SG}-; \mathrm{O}-$.

Cf., however, SG pẹćini 'di pietra', na péćina míza 'una panca di pietra'; $\mathrm{G}$ péćini ‘di pietra'; O pẹ́njćine 'di pietra', na pẹnjćina táwla 'una tavola di pietra'.

Remark: The suffix actually used is -in-, like in stárdina s.v. strdníca, both adjectives derived from feminine substanives ending in a consonant.

(1. pečíca) '= kamenček', 'das Steinchen'. II: 17.

picíca 'kamešek' (BdC 1875: 99)

SG pićịca 'pietrina'; O paćịca 'pietrina'.

pečljìv 'kummervoll'. $I I: 18$.

$\mathrm{SG}-; \mathrm{O}-$.

Cf., however, SG invilán -ịna 'avvilito'.

pečnàt 'steinern': pečnato srce. II: 18.

$\mathrm{SG}-; \mathrm{O}-$.

Cf., however, SG nọ pẹćine sárce 'un cuore di pietra'; O to pẹnjćine sárce 'cuore di pietra'.

péklọ ‘= pekel'. II: 20.

pẹkộ 'ad' (BdC 1875: 76).

SG paklọ 'inferno'; G puklọ 'inferno'; O paklọ 'inferno'; $\mathrm{S}$ paklọ/poklọ 'inferno'.

(persógelj) persoglji 'der Samenhanf'. II: 25.

$\mathrm{SG}-; \mathrm{O}-$.

1. pệsica dem. pesa 'der Mangold'. II: 26.

$\mathrm{SG}-; \mathrm{O}-$.

Cf., however, SG zéje 'bietola selvatica'.

pẹ́stiti if '= pestovati'. II: 28 .

SG péstit: péstit noga otroké 'far dire a un bambino quello che non dovrebbe dire secondo i suoi famigliari'; $\mathrm{O}$-.

Cf., however, O nastét tu-w péste 'portare nelle mani'.

(pęetred) petredi, petredov. II: 32 .

$\mathrm{S} \rightarrow \mathrm{P}$ paterdú 'fünfzig' (BdC 1895: 104). 
SG patardú 'cinquanta'; G patardú 'cinquanta’; O patardúw 'cinquanta’; S patardúw 'cinquanta'.

Remark: For the form variant petredi there is no Resian basis.

pę̂tredni '= petdeseti'. II: 33 .

SG -; O -.

(1. pîčka) ‘der Kürbis-, Zwetschken-, Pfirsichkern'. II: 34.

SG pátku 'nocciolo, seme (della zucca, della susina, della pesca ecc.)'; O -.

Cf., however, O kopit.ta 'seme (della zucca, della mela, della pesca)'; S kopịta 'seme (della zucca o del frutto)'.

(píla) 'das Rückgrat'. II: 37.

SG píla 'sega'; O pịla 'sega; addentatura della spina dorsale'; S pịla 'sega; spina dorsale'.

(plâhta) 'das Segeltuch'. II: 46.

SG pláhta 'straccio di una certa stoffa ruvida, iuta'; O pláxta 'tela che si mette nella gerla per trasportare letame'; S pláhta 'tela di iuta per trasportare foglie secche o fieno'.

plàn 'der Schwung': ogenj je planil v enem planu do strehe. II: 48. SG -; O -.

(plániti) pf 'auflodern'. II: 48.

$\mathrm{SG}-; \mathrm{O}-$.

plę̂ta 'die Falte'; 'die Runzel'. II: 58.

SG pléta 'piega; ruga'; O pléta 'ruga'; S pléta 'ruga'.

pletę́nčič 'das Handkörbchen'. II: 58.

G pletenčèć 'Körbchen' (BdC 1895: 551, 123)

SG plitinčáć -ị́ca 'cestino'; O platančéć -líća 'cestino'.

Remark: This lexeme is a diminutive of the one below.

(pletęenec -nca) 'das Handkörbchen'. II: 58.

platanàc 'kozin(k)a' (BdC 1875: 23).

SG platanác -ncé 'cesto’; G platanéc -ncá 'cesto; O platanác -ncá 'cesto’; S platanác -ncá 'cesto'.

(pleténje) ‘= srobot', 'die Waldrebe (clematis)'. II: 59.

SG platanjẹ 'convolvolo, Convolvulus sepium'; G plitinjẹ 'convolvolo, Convolvolus sepium'; O platanjẹ 'convolvolo, Convolvulus sepium'; S platanjẹ 'convolvolo, Convolvulus sepium?

2. pòč 'der Brunnen'. II: 72.

SG pọ́c - $a$ 'pozzo'; O poóč - $a$ 'pozzo'.

Remark: Cf. also SG pác - $a$ 'pozzo'; S péč -ačá 'pozzo'.

131

(poljúben -bna) 'zahm': poljubna toca 'eine Katze, die sich streicheln lässt'. II:

SG pujụ́ban -bna 'docile' (di animali); 'buono' (di bambini); G pujụban -bna 'docile'; $\mathrm{O}$-; S pojụben -bna 'mansueto'.

Cf., however, O krộtåk -tka 'mansueto'.

(poljúbiti) pf 'abstreicheln, liebkosen'. II: 131.

SG pujụbit -in pf 'accarezzare'; G pujụ́bit 'accarezzare'; O pojụbet -en pf 'accarezzare'; S pojụ́bit 'accarezzare'. 
$\mathrm{SG}-; \mathrm{O}-$.

(poljúbljati) if 'streicheln'. II: 131.

poromóniti se pf '= pogovoriti se'. II: 161.

SG se purumunát -ịn pf 'spiegarsi, chiarirsi'; O sæ poromonét -ịn pf 'parlare assieme'.

(pộzad) 'der Herd' "pozed". II: 202.

SG -; G pózet 'focolare'; O pọ́zet -ada 'buco nel pavimento per fare fuoco', ni so paklé boádnek ta-nu w pọ́zadu 'hanno cotto una focaccia nel focolare'.

207.

požledíti se -ím se pf 'sich mit Eis überziehen': drevje se je požledilo. II:

SG se poladanẹ́t -i 'coprirsi con ghiaccio'; O sæ poladanệt -í pf 'coprirsi con ghiaccio', ko to sæ poladaní, to puzí 'quando tutto è coperto con ghiaccio, si scivola'.

Remark: See also žlệdnat.

(prásčič) 'die Assel'. II: 210.

G Npl praščići 'Ferkel' (BdC 1895: 215).

SG prášćić - $a$ 'maialino'; O prášćeć -ića 'maialino'; S prášćić 'maialino'.

Cf., however, O bábica 'asello'.

$\mathrm{SG}-; \mathrm{O}-$.

prečígati pf '= prestreči’, 'lauernd zuvorkommen'. II: 222.

2. préjja 'die Schnalle (an Kleidern, Schuhen)' (Vestnik). II: 239.

Npl preje 'die Schnallen' (Dobrovský 1834: 120).

SG -; O préa 'fibbia'.

Cf., however, SG rịnća 'fibbia'; G rịnća 'fibbia', S rịnća 'fibbia'.

(prepẹ́kati -kam/-čem) if prepekati se 'besorgt sein, sich ängstigen, wehklagen'. II: 263.

$\mathrm{SG}-; \mathrm{O}-$.

Cf., however, O skarbẹt -ịn if 'preoccuparsi', máte na rụde skarbị za své otrokæ̉ 'la madre è sempre preoccupata per i suoi figli'.

(prepelíca) 'der Schmetterling'. II: 263.

pripilíca 'babočka' (BdC 1875: 25).

SG pripilíca 'farfalla'; O prapalíca 'farfalla'.

prgíšča 'die Handvoll'. II: 296.

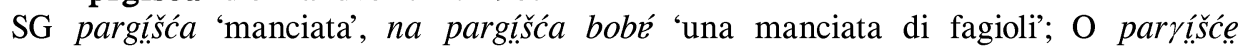
'manciata non piena'.

ràd '= dosti'. $I I: 370$.

rât 'dovol'no' (BdC 1875: 33).

SG rát 'abbastanza', se díwa gnúj, da prídi sáta rát 'si mette letame, affinché venga molta frutta'; O rát 'sufficiente', ko a sæ poromonịn, tadéj to rát 'quando finisco di parlare, basta'.

rádẹ 'gern'. II: 370.

SG ráde 'gern' (BdC 1895: 4).

SG rédi 'con piacere', ja rédi právin ziz tabó 'parlo volontieri con te'; $\mathrm{G}$ ráde 'con piacere', ni jín ráde mísu 'non mangio volontieri la carne'; O ráde 'con piacere', a ráde romonịn po rozoánskex 'parlo resiano con piacere'. 
(razdẹjáti -dệjem) pf razdejati obleko 'aufknöpfen'. II: 381.

riždât 'rastvorit', raskryt" (BdC 1875: 108).

SG rižđát -ždín pf 'stendere; togliere' riždẹj klubụ́k 'togliti il cappello'; O riždát, -ždín pf 'stendere; mettere via', rižđát sẹ́nu 'stendere il fieno'.

Cf., however, O odát క̌ụ̂po 'sbottonare la giacca'.

2. rẹpînje 'der Rübsamen, der Reps (brassica napus)'. II: 419.

$\mathrm{SG}-; \mathrm{O}-$.

Cf., however, SG rẹpa 'rapa'; O rẹ́pa 'rapa'.

(romộn) '= govor', 'die Sprache' rozeanski romon 'der resianische Dialect' (Letopis). II: 437.

Lp romonénj 'Aussprache' (BdC 1895: 33), G rumunínje 'Sprechen' (BdC 1895: 214).

SG romonénj -inja/-énja/-Énjaga 'parlata; modo di parlare', te bíski romonénj 'la parlata di San Giorgio; O -.

Cf., however, SG rumunínjẹ 'parlata'; O romonínjẹ 'parlata; modo di parlare', nášse romonínje to rozoánske 'la nostra parlata resiana'.

Remark: Caf (1852: 318) also has Npl romoni in the meaning 'conversations'. It should furthermore be noted that the lexeme romonénj is not judged to be a well-formed item by several informants. On the other hand, rumunínje etc. is generally accepted by them.

$\mathrm{SG}-; \mathrm{O}-$.

(romonệti -ím) if '= govoriti'. II: 437.

Remark: Apart from the deverbative SG rumuninje, O romoninje mentioned above there exists no form that could be analysed as deriving from a verb in -ẹti (Resian $-e(t)$. See also below.

romọ́niti -ộnim if '= govoriti'. II: 437.

$\mathrm{P}$ rumunèt 'sprechen' (BdC 1895: 46).

SG rumunát -ịn if 'parlare'; $\mathrm{G}$ rumunśt -ịn if 'parlare'; O romonét -ịn if 'parlare'; $\mathrm{S}$ romonát -ịn if 'parlare'.

sẹ́dniti, sệdnem pf '= sesti'. $I I: 465$.

sédnut 'sest" (BdC 1875: 82).

SG sédnut -nẹn pf 'sedersi'; G sédnut 'sedersi'; O sédnut -nąn pf 'sedersi', ko sédnąn, ćon sæ spočét 'quando mi siedo mi riposerò̀; $\mathrm{S}$ sédnot 'sedersi'.

See esej.

sej '= ta', 'dieser', sej - tej 'dieser - jener'. II: 465.

sfrlęénec -nca 'ein Butzel von Haarlocken'. II: 475.

$\mathrm{SG}-; \mathrm{O}-$.

sîrčje ‘= koruznica', ‘das Kukuruzstroh'. II: 480.

SG sirčjẹ 'stelo secco del granoturco'; O sirčjẹ 'paglia di granoturco'.

sklęti, skółnem pf 'verfluchen, verwünschen'. II: 489.

SG sklét/skúlnut, skúlnẹn pf 'sgridare', ni so me skúlnuli 'mi hanno sgridato'; O

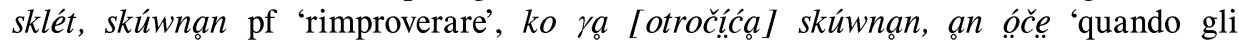
faccio un rimprovero, piange'.

Cf., however, SG moladíwat 'maledire'; O maladśt 'maledire', blaštamát 'bestemmiare'; S blaštamét 'bestemmiare'. 
$\mathrm{SG}-; \mathrm{O}-$.

sklínjati -am if impf. ad skleti (skolnem) 'verwünschen, verfluchen'. II: 489.

Cf., however, SG klét, kúlnẹn if 'sgridare', kúlnẹn me školérje, ki ni nịmajo wojo me puslụ́šat 'sgrido i miei scolari perché non mi vogliono ascoltare'.

3. skúta 'der Topfen, der Quark, der Streichkäse (die käsigen Bestandtheile der Milch, nach Abscheidung der Molken)'. II: 502.

G skụta 'Quark' (BdC 1895: 196).

SG skụ̂ta 'ricotta'; O skụta 'ricotta'.

2. slẹzeníca 'das Schneewasser'. II: 510.

SG - ; O slazanịca 'acqua di neve sciolta'.

slota 'regnerisches Wetter, Regen mit Schnee' II: 513.

SG slọta 'cambiamento di tempo', ni čụjajo slọto 'sentono il cambiamento di tempo'; G slọta 'periodo di cattivo tempo'; O slọta 'temporale'; S slọta 'pioggia che continua per parecchi giorni', te valị̂ke slọte 'le grandi pioggie'.

slotati if 'stürmen und regnen'. II: 513.

SG -; O slotát -á if 'esserci un temporale', to škụ́r, to će slotát 'fà buio, ci sarà un temporale'.

$\mathrm{SG}-; \mathrm{O}-$.

slotav 'regnerisch'. II: 513.

Cf., however, Lp slọtan -tna 'temporalesco'.

smlęed 'bleich, gelb (von der Gesichtsfarbe)'. II: 519.

smljidy [no translation] (Sreznevskij 1841: 154).

SG smlít - $d a$ 'pallido (della faccia, delle mani)'; G smlít 'pallido'; O smlít -da 'pallido (della pelle umana)', smlída víšta 'una faccia pallida', smlídæ rókæ 'mani pallide'; $\mathrm{S}$ smlít -da 'pallido'.

$\mathrm{SG}-; \mathrm{O}-$.

smọ́da ‘= smod'. II: 519 .

(smolíka) 'der Wacholder'. II: 520-521.

SG smulíka 'možževel'nik' (BdC 1875: 96).

SG smulị̂ka 'ginepro'; O smolị̂ka 'ginepro'.

2. smúkati -kam/-čem if 'schluchzen, winseln'; 'schwatzen'. II: 524.

SG smụkat -čẹn if 'singhiozzare, piagnucolare'; O smụ́kat -kan/-čan if 'soffiarsi il naso', to smụ́če tu-w nọsu 'il naso fa rumore'.

(sóditi) if 'herrichten': posteljo, mizo soditi. II: 529.

SG sódit -in if 'condire'; O sódet -en if 'condire; preparare', sóden soláto 'condisco l'insalata', sóden kówo 'preparo il letto'.

Cf., however, SG pasójat -an 'preparare (il letto)', ja si pasójan kówo 'mi sto facendo il letto'.

(sộk) 'ein zubereitetes Gemüse, die Zuspeise'. II: 531.

O súk 'Kraut' (BdC 1895: 402).

SG súk -ọka 'crauti'; O súk -ộka 'crauti', te rẹpine súk 'crauti di rapa'; $\mathrm{S}$ súk 'crauti'.

(spomîn) 'die Erforschung des Gewissens'. II: 549.

spomágn [no translation] (BdC 1913: 29).

SG spománj -a 'riccordino'; O spománj 'riccordo'. 
Cf., however, SG se spomanụt -ẹ́n pf 'cercare di riccordarsi' (come preparazione alla confessione).

(stópa) 'die Fußstapfe, die Fußspur'. II: 579.

stópa 'stupa' (BdC 1875: 24).

SG stópa 'orma'; O stópa 'orma; passo'.

strdníca '= medica', 'der Meth'. II: 586.

$\mathrm{SG}-; \mathrm{O}-$.

Cf., however, O ta stárdina wọ́da 'bevanda non alcolica fatta con miele'.

(stûlek -1ka) 'krause Locken'. II: 597.

$\mathrm{SG}-; \mathrm{O}-$.

Cf., however, SG čafarónaste láse 'capelli crespi'; O čafarúnastæ lásæ 'capelli crespi'.

(stúliti) pf stuljeno hoditi 'gebückt, gekrümmt einher gehen'. II: 597.

SG stújẹn 'ristretto' wudát stújẹn 'andare un po' curvo'; G stụ́en -ana 'inchinato'; O

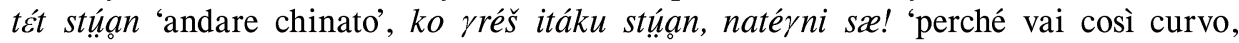
raddrizzati!'

sûj 'Schaff, Sechter'. II: 600-601.

P sój 'Schaff, Waschbecken' (BdC 1895: 68).

SG sứj-a 'mastello'; G sój 'mastello'; O sój -ụa 'mastello'.

Cf., however, SG ćáča 'ramaiolo'.

svìk '= svaljek'. II: 615 .

$\mathrm{SG}-; \mathrm{O}-$.

Cf., however, SG no závite 'qualcosa arrotolata'.

ščè "šttje". II: 618.

šće 'eščë' (BdC 1875: 24).

SG šcé 'ancora'; O šćé 'ancora'; S šćé 'ancora'.

ščírovec -vca 'das Geschwür' (Vestnik). II: 621.

G šćírovac 'eine Hautausschlag' (BdC 1895: 198).

SG -; G šćírovac -uwca 'foruncolo; O šćíravac -uwca 'foruncolo'.

$\mathrm{SG}-; \mathrm{O}-$.

šùj ‘= lev', 'link’. II: 649.

Cf., however, (hûd).

Remark: The nominal phrase G ná te šúnini kráj 'nach der linken Seite' (BdC 1895:

187) is later on corrected into [ná te] šúnčni [kráj] 'nach der Sonnen-Seite' (BdC

1895: 560, 601).

tà ' $=$ tja'. $I I: 653$.

SG tén, té 'di là; O itá, táa 'di là'; S tá 'là.

tâsa 'ein Haufen aufgeschichtetes Holz, Heu oder Garben'. II: 656.

G na tása dáru 'ein Maß, eine Lage, eine Schicht Holz' (BdC 1895: 155).

SG tása 'catasta', na tása lóduw 'una catasta di legna'; O tása 'catasta', na tása dórw 'una catasta di legna', na tása brúušć 'una catasta di ramoscelli secchi'.

tintína 'die Maultrommel'. II: 669.

$\mathrm{SG}-; \mathrm{O}-$.

Cf., however, O tintịn 'corda del violino'. 
tintínati if 'auf der Maultrommel spielen'. II: 669.

$\mathrm{S} \rightarrow \mathrm{P}$ tintinàt 'läuten' (BdC 1895: 347).

$\mathrm{SG}-; \mathrm{O}-$.

Cf., however, SG tintinét -án if 'scampanare, battendo con campanelle contro le campane del campanile'; O tintinát -á if 'scampanare', sæ tintiná ko so fjẹsstæ 'si scampana quando ci sono le feste'.

(tōca) '= mačka' "toća". II: 673 .

túca 'koška' (BdC 1875: 75).

SG túca 'gatto (in senso generale)'; G túca 'gatto'; O túca 'gatto' (in senso generale); S túca 'gatto'.

točák 'der Kater'. II: 673.

$\mathrm{SG}-; \mathrm{O}-$.

Cf., however, SG máčak 'gatto maschile'; O máčak -aka 'gatto maschile'.

tọ́čica 'das Kätzchen'. II: 673.

Ndu túčice 'Kätzchen, Katzen’ (BdC 1895: 369).

SG túčica 'gattino'; G túčica 'gattino'; O túčica 'gattino'.

$\mathrm{SG}-; \mathrm{O}-$.

togotáti se if 'trauern'. II: 674

1. tọ́hniti if 'dumpfig, muffig werden'. II: 674 .

SG tófnẹn -ana 'ammuffito'; O tófnan -ana 'ammuffito'.

Cf., however, SG se smufavệt -í pf 'ammuffire', sẹ́r se smufavél 'il formaggio è ammuffito'.

Remark: The existence of a verb from which tófnen etc. would be a derived form is explicitely denied by the informants.

(tọ́žen -žna) '= otožen', 'traurig'. II: 680 .

tôžen 'grustnyj' (BdC 1875: 54).

SG tóžan -žna 'triste'; G tóžan -žna 'triste'; O tóžan -žna 'triste'; S tóžen -žna 'triste'.

tọ́žnost 'die Traurigkeit, die Betrübnis'. II: 680.

toshnost 'malinconia' (Matičetov-Perusini 1955-56: 86).

SG -; O tóžnust (arch.) 'tristezza'.

(trếbuh) 'die Kuttelflecke' "trebuhove". II: 685.

trîbuh 'život' (BdC 1875: 11).

$\mathrm{SG}-; \mathrm{O}-$.

Cf., however, SG tríbu -ụha 'pancia', Npl trịpe 'trippa'; G tríbuh 'pancia'; O tríbux, -úxa 'pancia', Npl trípæe 'trippa'; S tríbo 'pancia'.

(trg) dober $\operatorname{trg}$ '= dober kup', 'wohlfeil'. II: 689.

$\mathrm{S} \rightarrow \mathrm{P}$ dóber tér 'billig' (BdC 1895: 346).

SG dóbar tárk 'buon mercato’; G dóbar tárh 'buon mercato'; O dóbąr tárx 'buon mercato'; S dóbar tár 'buon mercato'.

(trọ́ba) 'die Stiefelröhre'. II: 694.

$\mathrm{SG}-; \mathrm{O}-$.

(trộmba) 'das Brunnenrohr'; 'das Stiefelrohr'. II: 696.

O trúmba 'Gosse' (BdC 1895: 284).

SG trómba 'fontana'; G trúmba 'fontana'; O trúmba 'tubo; fontana'; S trúmba 'fontana'. 
trúpati -pam/-pljem if 'zerbrechen, zerstören': trupati zid. II: 700.

SG trúpat -an if 'bussare'; $\mathrm{O}-$.

Cf., however, SG vijét dọlu din mír 'distruggere un muro'; O trụ́mpat -an if 'battere con le piedi'.

(tûlast) 'röhrenformig, hohl'. II: 702.

$\mathrm{SG}-; \mathrm{O}-$.

Cf., however, O tứrlast -asta 'tarmato'.

Remark: See also turláti.

(1. tûlec -lca) 'der Hutgupf'; 'etwas Zusammengerolltes'. II: 702.

SG túlac -aca 'nuca'; O túlac 'il dietro della testa, nuca'.

tulîn 'der Wasserwirbel'. II: 702.

$\mathrm{SG}-; \mathrm{O}-$.

Cf., however, SG se naréja kotál 'si forma un mulinello; O wọ́da naréa kotów 'lacqua fà il mulinello?.

Remark: S Tulin TOP, indicating a point with a small waterfall in a brook, might be identical with this item (Dapit 1995: 200). $\mathrm{SG}-; \mathrm{O}-$.

tulíniti se if 'sich wirbeln' (o vodi). II: 702.

(2. túliti) if tuliti se 'sich wirbeln': voda se tuli; tuliti se h komu 'sich an jemanden schmiegen'. II: 702 .

SG se túlit -in if: se tụ́lin za mrázon 'mi viene la pelle d'oca per il freddo'; O sæ túlet -en if 'restringersi', otrộk an sæ tụ́le ta-x mátare 'il bambino si rannichia accanto alla madre'.

Cf., however, SG se stískat -šćẹn if 'stringersi contro qualcuno', se stískajo wkụp 'si stringono assieme'.

(turláti -âm if 'durchbohren, durchbeissen') molj turla in vrta. II: 703.

SG turlét -án if 'tarlare' (soltanto di legno); O turlát -á if 'rodere' (di tarme).

Cf., however, SG tárme snádajo ublič̣̂le 'le tarme mangiano i vestiti’.

tûrlej ' $=$ molj’. II: 703.

$\mathrm{SG}-; \mathrm{O}-$.

Cf., however, SG tárma 'tarma'; O tárma 'tarma'.

učígati pf 'erlaueren, erspähen'. II: 707.

$\mathrm{SG}-; \mathrm{O}-$.

udrístati se pf 'infolge Durchfalls sich beschmutzen'. II: 710.

SG se wdrịstat -šćẹn pf 'sporcarsi avendo la diarrea'; O -.

Cf., however, O sæ zdrịstat -šćan pf 'sporcarsi avendo la diarrea'.

ûj '= ujec'. II: 715 .

wòj 'djadja' (BdC 1875: 57).

SG wụj -a 'zio'; G wój -ụja 'zio'; O wój -ụja 'zio, prozio; S wój 'zio’.

ukísniti pf 'sauer werden': moka ukisne. II: 717.

$\mathrm{SG}-; \mathrm{O}-$.

Cf., however, O sæ skwáset -e pf 'diventare acido'.

(ulęči, ulęežem) pf 'sich krank niederliegen'. II: 719.

SG wlégnut -ẹn pf 'sdraiarsi ammalato', ko wlégnẹn, se spútin anu prijája pá tréši- 
$k a$ 'quando mi sdraio ammalato, sudo e viene anche la febbre'; O wlérnut -an pf 'sdraiarsi ammalato', ko wlérnạn, ćon stát boẹ 'quando mi sdraio ammalato, starò meglio'.

(ulęgniti) pf ulegnilo mi je v želodec 'ich bekam Magenbeschwerden'. II: 719.

$\mathrm{SG}-; \mathrm{O}-$.

Cf., however, SG ja man ta-po štómide 'ho disturbi gastrici'.

ulẹ́niti se pf ulenjena koza '= koza, ki se je ulevila'. II: 720 .

SG se odlénit $-i$ pf 'cambiare pelle', kozé je odlénjana 'la capra ha cambiato pelle; O sæ oblẹnet $-e$ pf 'perdere i capelli, le penne', mu sæ oblénila wsá rláwa 'è diventato tutto calvo', kozá na oblénjana 'la capra ha cambiato pelle'.

(ulízniti) pf moja glava se mi je uliznila 'ich habe die Haare verloren'; uliznjena koza '= ulenjena koza'. II: 720 .

$\mathrm{SG}-; \mathrm{O}-$.

Cf., however, ulệniti se.

ulja '= gos'. $I I: 720$.

$\mathrm{SG}-; \mathrm{O}-$.

Cf., however, O óća 'oca'.

uljen '= ulj'. II: 720 .

P wújan 'ulej' (BdC 1875: 36).

SG wúlin - $a$ 'arnia'; $\mathrm{G}$ wụjen -ana 'alveare'; $\mathrm{O}$ wúan -ana 'arnia'; $\mathrm{S}$ wújẹn -ana 'arnia'.

(úm) 'der Zorn', na umu biti 'zornig sein'. II: 721.

ty sè naûmo 'ty serdišsja' (BdC 1875: 14).

SG wứm - $a$ 'rabbia', si na wụ́mu 'sono arrabbiato'; G wứm 'rabbia', ni so ha méli wứm 'erano arrabbiati con lui'; O wứm 'rabbia', a man wụm 'sono arrabbiato’; S wúm 'bile; rabbia'.

(úmiti) if umiti se 'zornig sein, zürnen'. II: 724.

umat 'odiare' (Matičetov-Perusini 1955-56: 86).

SG -; O -. In a song, pre3sg wúma 'jezi se' is attested (Matičetov 1998: 59).

Cf., however, O sæ rizdrážet 'arrabbiarsi'.

SG -; O -.

upírav 'widersetzlich, widerspenstig'. II: 727.

(ûren -rna) úren '= goden', 'flügge': uren ptič. II: 730.

SG -; G wụrenn 'uccello giovane nel suo nido'; $\mathrm{O}$-.

Cf., however, SG an je wứra za listót 'è pronto per volare'.

úrenčič 'ein flügger Vogel'. II: 730.

SG wứrančić - $a$ 'uccello giovane capace di volare'; G Npl wúrančići 'uccello giovane capace di volare'; O wứrančeć -ića 'uccello giovane capace di volare'.

urọ́biti pf '= posekati': urobiti drevo. II: 731 .

SG wróbit -in pf 'abbattere'; $\mathrm{G}$ wróbit árbule 'tagliare alberi'; $\mathrm{O}$ wróbet -en pf 'tagliare (alberi)'.

(1. usọ́diti) pf '= zabeliti, začiniti (jed)'. II: 734 .

Lp osódit lidrèk, soláto 'radicchio (Cichorien-Salat), Salat anmachen' (BdC 1895: 32).

SG wosódit -in pf 'condire', wosódit minjẹsstro 'condire la minestra'; G si usódila dado anu olio 'ho condito con un dado e con olio'; O osódet -en pf 'condire'. 
$\mathrm{SG}-; \mathrm{O}$-.

uvę́dniti pf ‘= uveniti'. II: 741 .

Cf., however, SG se wpéhnut -ne pf 'appassire'; O sæ wpáxnut pf 'appassire'; S 1-ptc fsg se wpénula 'appassire'.

(uvrẹ́diti) pf uvrejen infolge des Überhebens innerlich beschädigt'. II: 742. ppp urijen, sè urídit 'wenn man sich verhoben hat' (BdC 1895: 623).

SG se wrídit -in 'sforzarsi'; O sæ wrídet -en pf 'farsi male sforzandosi'.

(vâkati) if vaka se mi 'es ist mir zum Erbrechen, es ekelt mich'. II: 746.

G uákat 'brechen' (BdC 1895: 161).

SG wákat -čẹn if 'vomitare', to mi parája za wákat 'mi viene da vomitare'; G wákat 'vomitare'; O wákat 'vomitare' non-O; $\mathrm{S}$ to wáče 'mi viene la nausea'.

Cf., however, O bjuwát 'vomitare', to mi parxáa za bjuwát 'mi viene da vomitare'; $\mathrm{S}$ bjuwśt -õn 'vomitare'.

vę́nahti ‘= božič'. II: 759 .

O Gpl v'ínahat 'Weinachten' (BdC 1895: 277).

SG Vinahti 'Natale'; G Vinahti 'Natale'; O Vinaxte - uw 'Natale'; S Vinati -et 'Natale'.

1. vlážen -žna 'lau (vom Wetter)'. II: 777.

P sẹno je wlážno 'das Heu is feucht' (BdC 1895: 67).

SG wlážan -žna 'tranquillo' (del tempo); O wlážan -žna 'mite, umido, senza vento' (del tempo); S wlážan -žna 'umido'.

Cf., however, SG womárast - $a$ 'tiepido' (del tempo), S omõrest 'tiepido'.

(vozíca) 'der Schiebkarren'. II: 788.

G ûzica 'Schubkarren' (BdC 1895: 423).

$\mathrm{SG}-; \mathrm{O}-$.

Cf., however, SG karjọla 'carriola', uzáć -ḷća 'carretto'; O karjọla 'carriola', wozéć, -ḷća 'carro' (dim.).

(vrẹcče) 'der Hodenbeutel'. II: 794.

wrécée 'měšok' (BdC 1875: 25).

SG wrẹcée 'sacco'; O wrẹcée 'sacco'.

(zadẹjáti -dẹ́nem) pf suknjo zadejati '= zapeti'. II: 823.

SG zadát -dín pf 'chiudere'; O zađát -dín pf 'chiudere'.

zádẹšnji 'hinterig'. II: 823.

$\mathrm{SG}-; \mathrm{O}$-.

Cf., however, SG zádnji 'ultimo, posteriore'; O zádnje 'ultimo, posteriore'.

zadr̂ga 'die Verknüpfung (mittels eines Knotens)'. II: 826.

$\mathrm{SG}-$; O -.

(zadŕgniti) pf vrvco zadrgniti. II: 827.

SG -; O -.

Cf., however, SG zavézat wárco 'annodare il filo'.

zadrúskniti pf 'zuschlagen': vrata zadruskniti. II: 827.

SG zadrụ́šnut -ẹn pf 'sbattere', zadrụśnut dúri 'sbattere la porta'; O -.

Cf., however, O zdrụkknut -an pf 'sbattere'.

zadrúščiti pf '= zadruskniti'. II: 827.

SG -; O -. 
Cf., however, SG zdrušćét -án pf 'mandare in frantumi'; O zdrušćát -án pf 'mandare in frantumi'.

$\mathrm{SG}-; \mathrm{O}-$.

zafŕniti pf '= zafrkati', zafrnjeni lasje 'gekraustes Haar'. II: 828 .

Cf., however, SG zafárjani lásji 'capelli non pettinati'.

zafr̂njenik 'der Krauskopf'. $I I: 828$.

$\mathrm{SG}-; \mathrm{O}-$.

Cf., however, SG zafórjanik 'persona dai capelli non pettinati'.

(zagníti) “zagnuti” 'umbiegen'. II: 830.

SG zagnụt - $a$ 'curvo', jté an gré zagnụt 'lui va curvo'; O zarnót -ẹn pf 'chinare', ąn stojị zarnút 'sta curvo'.

(zagrábiti) pf '= zagrebsti'. II: 832.

SG zagrábit -in pf 'egalizzare la terra con il rastrello'; O zarrábet -en pf 'raschiare con il rastrello'.

zagrâja 'die Verzäunung, der Zaun'. II: 832.

$\mathrm{SG}-; \mathrm{O}-$.

Cf., however, SG zagrádit -in pf 'dividere i campi'; O to zarráano 'il posto recintato', zarráda 'striscia di terra posta di traverso fra le porche del campo dove si seminano i fagioli' (Dapit 1998: 191).

$\mathrm{SG}-; \mathrm{O}-$.

zalèz zalez solnčni 'der Sonnenuntergang'. II: 847.

zamíriti pf '= zazidati', 'vermauern'. II: 853 .

SG zamírit -in "chiudere con un muro'; $\mathrm{O}-$.

zanę́ti -námem pf 'entzünden'. II: 857.

SG se zanitit -te pf 'autoincendiare'; $\mathrm{O}$ sæ zanitet -te pf 'divampare'.

SG -; O -.

zapréjiti pf 'zuschnallen'. II: 868.

Cf., however, SG zadát rịnćo 'chiudere la fibbia'; O zadát préo 'chiudere la fibbia'.

(zbrznẹ́ti) pf ogel zbrzni 'die Köhle wird zu Asche'. II: 905.

$\mathrm{SG}-; \mathrm{O}-$.

Cf., however, SG wógul se tlé anu parája pẹpẹl 'il carbone brucia lentamente e diventa cenere'.

zgôdnji 'Früh-, frühzeitig'. II: 916.

SG zgúdnji 'presto'; G zwúdnji 'presto'; O zwúdnje 'presto'.

(zîd) '= pozid', 'der Herd'. II: 920.

$\mathrm{SG}-; \mathrm{O}-$.

(zvâra) 'das Käsewasser'. II: 943 .

G Asg zwáro 'Molken' (BdC 1895: 187).

SG zwára 'siero'; O zwára 'siero', ko zwára zawrí, parxáa skúta 'quando il siero bollisce viene la ricotta'.

(žábica) ‘das Anhängeschloss'. II: 951.

SG -; O žábica 'rana' (dim.); 'piccolo rampone'.

Cf., however, SG žlós -ósa 'lucchetto’; O žlós -ósa 'lucchetto'. 
žímen 'das einzelne Rosshaar'. II: 962.

$\mathrm{SG}-; \mathrm{O}-$.

Cf., however, SG dláka wod konjé 'pelo di cavallo'; O ta konjộwja dláka 'pelo di cavallo'.

žímenje ‘das Rosshaar’. II: 962.

$\mathrm{SG}-; \mathrm{O}-$.

žlệdnat 'glatteisig': žlednato drevje. II: 968.

$\mathrm{SG}-; \mathrm{O}-$.

Cf., however, SG poledenẹt - $a$ 'coperto con ghiaccio'; G pulidinẹt 'coperto con ghiaccio'; O poladanẹt 'coperto con ghiaccio', pót e poladanẹta 'la strada è coperta con ghiaccio'; S ppp Nsgf poladanéta 'coperto con ghiaccio'.

Remark: SG poledenẹt is the third known attestation with pretonic /E/ [ə]. Considered together with the other two items, šenọ 'if not' and nevésta 'daughter-in-law', one starts to think that the $/ \mathrm{n} /$ is somehow responsible for this preservation of an unrounded mid vowel in pretonic position. $\mathrm{SG}-; \mathrm{O}-$.

žlẹdọ́ven -vna 'Schauerregen-': žledovno vreme. II: 968.

Prispelo oktobra 2000, sprejeto decembra 2000

Received October 2000, accepted December 2000

\section{Cafov prispevek k rezijanski leksikografiji}

Sodeč po prispevanem gradivu za Pleteršnikov slovar ima Oroslav Caf (1814-1874) velike zasluge za razvoj rezijanske leksikografije. Iz Cafovega slovarskega gradiva namreč izvira več kot 300 Pleteršnikovih iztočnic in mnoge izmed njih nam niso znane iz nobenega drugega vira.

Jezikoslovna dejstva kažejo na to, da Cafovo gradivo izvira iz Bile/San Giorgio. Standardizacija glasu $\grave{u} \mathrm{z} \hat{u}$ se namreč najlaže razloži z vplivom govora, $\mathrm{v}$ katerem

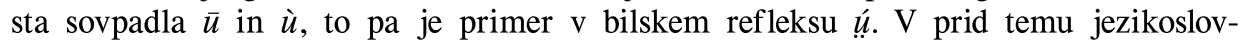
nemu argumentu govorijo tudi zgodovinska dejstva, na katera opozarja Matičetov. Približno tretjine besed iz Cafove zbirke danes na terenu ne moremo več potrditi. Razen primera vozíca, ki ga Baudouin de Courtenay navaja kot $\hat{z} z i c a$, jih ne najdemo niti v starejših virih. Ti primeri imajo za nadaljnjo jezikoslovno analizo omejeno vrednost, saj sta Pleteršnikovo »filtriranje « narečnih zapisov v standardizirane in Cafovo nagnjenje $\mathrm{k}$ etimologiziranju lahko zameglili njihovo dejansko narečno podobo.

\section{The Contribution of Oroslav Caf to Resian Lexicography}

To judge by the Resian lexical items in Pleteršnik for which Oroslav Caf (1814-1874) is credited, it may be said that his contribution to Resian lexicography is 
considerable. This goes for the sheer amount of the items, adding up to more than 300 , and for their choice, as many of them are not known from other sources.

Linguistic evidence strongly suggests that the dialect material originates from a native speaker of the Bila/San Giorgio variety of Resian. The standardisation of $\grave{u}$ as $\hat{u}$ can easiest be explained as stemming from a dialect in which $\bar{u}$ and $\grave{u}$ have merged, like in the Bila reflex $\underline{u}$. This evidence is corroborated by historical evidence brought forward by Matičetov. In about one third of the cases no modern attestations could be obtained for Caf's lexical items. Apart from the item vozíca 'der Schiebkarren', confirmed as ûzica by Baudouin de Courtenay, these items are not found in other sources either. This means that they could be of limited use for further linguistic analysis, as the "filtering" by Pleteršnik, who standardised the forms for the purposes of his dictionary, and Caf, who was prone to etymologise in his dialect transcriptions, may have obscured the actual underlying dialect form. 